\title{
Herbs and Spices- Biomarkers of Intake Based on Human Intervention Studies - A Systematic Review
}

\author{
Rosa Vázquez-Fresno ${ }^{1 *}$ (D), Albert Remus R. Rosana', Tanvir Sajed², Tuviere Onookome-Okome',
} Noah A. Wishart ${ }^{1}$ and David S. Wishart ${ }^{1,2}$

\begin{abstract}
Culinary herbs and spices have been used as both food flavoring and food preservative agents for centuries. Moreover, due to their known and presumptive health benefits, herbs and spices have also been used in medical practices since ancient times. Some of the health effects attributed to herbs and spices include antioxidant, antimicrobial, and anti-inflammatory effects as well as potential protection against cardiovascular disease, neurodegeneration, type 2 diabetes, and cancer. While interest in herbs and spices as medicinal agents remains high and their use in foods continues to grow, there have been remarkably few studies that have attempted to track the dietary intake of herbs and spices and even fewer that have tried to find potential biomarkers of food intake (BFIs). The aim of the present review is to systematically survey the global literature on herbs and spices in an effort to identify and evaluate specific intake biomarkers for a representative set of common herbs and spices in humans. A total of 25 herbs and spices were initially chosen, including anise, basil, black pepper, caraway, chili pepper, cinnamon, clove, cumin, curcumin, dill, fennel, fenugreek, ginger, lemongrass, marjoram, nutmeg, oregano, parsley, peppermint and spearmint, rosemary, saffron, sage, tarragon, and thyme. However, only 17 of these herbs and spices had published, peer-reviewed studies describing potential biomarkers of intake. In many studies, the herb or spice of interest was administrated in the form of a capsule or extract and very few studies were performed with actual foods. A systematic assessment of the candidate biomarkers was also performed. Given the limitations in the experimental designs for many of the published studies, further work is needed to better evaluate the identified set of BFls. Although the daily intake of herbs and spices is very low compared to most other foods, this important set of food seasoning agents should not be underestimated, especially given their potential benefits to human health.
\end{abstract}

Keywords: Food exposure biomarker, Metabolomics, Herbs, Spices, Diet

\section{Background}

Spices are the dried, pleasantly aromatic parts of the plants. More specifically, as defined by the Food and Drug Administration organization (FDA), spices are: "aromatic vegetable substances, in the whole, broken, or ground form, whose significant function in food is seasoning rather than nutrition" [1]. The main difference between a herb and a spice is that a spice comes from any part of a plant other than the leaves while a herb

\footnotetext{
* Correspondence: rosavfr@gmail.com

${ }^{1}$ Department of Biological Sciences, University of Alberta, Edmonton, AB T6G 2E9, Canada

Full list of author information is available at the end of the article
}

always comes from the leaves [1]. Spices typically come from the dried part of a plant such as buds, flowers (cloves, saffron); bark (cinnamon); root (ginger, turmeric); fruits/berries (cloves, chili, black pepper); or seeds (cumin) that contain volatile oils or aromatic scents and flavors $[1,2]$ (see Table 1 ). Most of the known herbs and spices originate from Mediterranean countries, the Middle East or Asia, and many have been used since ancient Egyptian and Roman times [3].

Herbs and spices have played, and continue to play, important roles as flavoring agents, food preservatives and medicines for centuries. Over the last few decades, research into their health benefits has 
Table 1 Scientific and common names of selected spices evaluated in this review

\begin{tabular}{|c|c|c|c|}
\hline Num. & $\begin{array}{l}\text { Name of } \\
\text { spice }\end{array}$ & Scientific name & $\begin{array}{l}\text { Part of } \\
\text { the plant }\end{array}$ \\
\hline 1 & Anise & Pimpinella anisum & $\begin{array}{l}\text { Seed/ } \\
\text { fruit }\end{array}$ \\
\hline 2 & Basil & Ocimum basilicum & Leaf \\
\hline 3 & $\begin{array}{l}\text { Black } \\
\text { pepper }\end{array}$ & Piper nigrum & Berry \\
\hline 4 & Caraway & Carum carvi & Fruit \\
\hline 5 & $\begin{array}{l}\text { Chili } \\
\text { pepper }\end{array}$ & $\begin{array}{l}\text { Capsicum annuum, Capsicum baccatum, } \\
\text { Capsicum chinense, Capsicum frutescens, } \\
\text { Capsicum pubescens }\end{array}$ & Fruit \\
\hline 6 & Cinnamon & Cinnamomum & Bark \\
\hline 7 & Clove & Syzygium aromaticum & Bud \\
\hline 8 & Cumin & Cuminum cyminum & Seed \\
\hline 9 & Dill & Anethum graveolens & $\begin{array}{l}\text { Leaf/ } \\
\text { seed }\end{array}$ \\
\hline 10 & Fennel & Foeniculum vulgare & $\begin{array}{l}\text { Leaf/ } \\
\text { seed }\end{array}$ \\
\hline 11 & Fenugreek & Trigonella foenum-graecum & Seed \\
\hline 12 & Ginger & Zingiber officinale & Root \\
\hline 13 & Lemongrass & Cymbopogon & Leaf \\
\hline 14 & Marjoram & Origanum majorana & Leaf \\
\hline 15 & Nutmeg & Myristica fragrans & Seed \\
\hline 16 & Oregano & Origanum vulgare & Leaf \\
\hline 17 & Parsley & Petroselinum crispum & Leaf \\
\hline 18 & Peppermint & Mentha $\times$ piperita & Leaf \\
\hline 19 & Rosemary & Rosmarinus officinalis & Leaf \\
\hline 20 & Saffron & Crocus sativus & $\begin{array}{l}\text { Stigma } \\
\text { (flower) }\end{array}$ \\
\hline 21 & Sage & Salvia officinalis & Leaf \\
\hline 22 & Spearmint & Mentha spicata & Leaf \\
\hline 23 & Tarragon & Artemisia dracunculus & Leaf \\
\hline 24 & Thyme & Thymus vulgaris & Leaf \\
\hline 25 & $\begin{array}{l}\text { Turmeric } \\
\text { (curcumin) }\end{array}$ & Curcuma longa & Root \\
\hline
\end{tabular}

increased significantly, as many herbs and spices are known to possess properties associated with reducing the risk of developing chronic diseases. In particular, some of the potential health benefits of herbs and spices include conferring protection against cardiovascular disease, neurodegenerative conditions, chronic inflammation, cancer, obesity, and type 2 diabetes [414]. A number of herbs and spices have also been noted for their strong antioxidant, anti-microbial, and anti-inflammatory properties $[4,7,15]$. Moreover, the flavoring properties of many herbs and spices tend to reduce the use of salt as a flavoring agent (i.e., reduced sodium intake) which has additional cardiovascular health benefits [16].
Most of the positive health effects of herbs and spices towards preventing or ameliorating chronic diseases such as cancer, cardiovascular disease, arthritis, and neurodegeneration appear to be mediated through the direct action of their constituent phytochemicals (particularly polyphenols or polyphenol breakdown products) targeting specific receptors or enzymes involved in various anti-inflammatory pathways or immune responses [10]. Herbs and spices (especially in their dried form) contain high levels of polyphenols [6] and other physiologically active phytochemicals. The predominant class of polyphenols found in herbs and spices are the phenolic acids and flavonoids (mainly flavones and flavonols) [17]. Relative to other polyphenol-rich foods such as broccoli, dark chocolate, red, blue and purple berries, grapes or onions-herbs and spices generally contain somewhat higher levels of these compounds. For instance, oregano has $935.3 \mathrm{mg}$ of total phenolic content per $100 \mathrm{~g}$ of fresh weight (FW) in the fresh form (F) while in the dried form (D) has $6367 \mathrm{mg} / 100 \mathrm{~g}$. Similarly, high polyphenolic levels are seen in rosemary $[1082.4 \mathrm{mg} / 100 \mathrm{~g}(\mathrm{~F})$ vs. $2518 \mathrm{mg} / 100 \mathrm{~g}$ (D)], thyme $[1173.28 \mathrm{mg} / 100 \mathrm{~g}$ (F) vs. $1815 \mathrm{mg} / 100 \mathrm{~g}$ (D)], and parsley [89.27 mg/100 g (F) vs. $1584 \mathrm{mg} / 100 \mathrm{~g}$ (D)]. Likewise, cloves have $16,047.25 \mathrm{mg} /$ $100 \mathrm{~g}$, cinnamon $9700 \mathrm{mg} / 100 \mathrm{~g}$, and turmeric $2117 \mathrm{mg} /$ $100 \mathrm{~g}$ (all FW). In contrast, other non-herbs and foods such as dark chocolate contain $1859.8 \mathrm{mg} / 100 \mathrm{~g} \mathrm{FW}$, while raw blackcurrants contain $820.6 \mathrm{mg} / 100 \mathrm{~g}$ FW and broccoli just $198.6 \mathrm{mg} / 100 \mathrm{~g}$ FW.

Polyphenols, terpenoids, and other spice-derived alkaloids (such as capsaicinoids) are also known to possess antibacterial, antiviral, and antifungal properties [18]. This is one reason why herbs and spices are so frequently used as preservative agents in food [19]. The antimicrobial properties of herbs and spices have been attributed to their unique volatile oils and oleoresins [20]. For instance, comparative studies involving cloves, cinnamon, oregano, rosemary, sage, and thyme showed that thyme oil was particularly active against Aeromonas hydrophila - a pathogen widely distributed in the environment, domestic animals, and food [21]. Likewise, essential oils found in thyme, oregano, mint, cinnamon, and cloves were found to possess strong antibacterial properties against several food-borne bacteria and fungi [22].

The number of herbs with known or potential anti-inflammatory activity is quite significant. Of the 25 herbs and spices analyzed in this review, 21/25 (84\%) had at least one published study supporting an anti-inflammatory finding. The spices that are most frequently identified as having anti-inflammatory effects are thyme, oregano, rosemary, sage, basil, mint, turmeric, dill, parsley, cinnamon, clove, nutmeg, lemon grass, ginger, chili pepper, fenugreek, and pepper [4, 23]. Many of the anti-inflammatory compounds found in 
herbs and spices, such as curcumin, gingerol, and capsaicin, appear to operate by inhibiting one or more of the steps linking pro-inflammatory stimuli with cyclooxygenase (COX) activation [10]. While the mechanisms behind some of the health benefits in herbs and spices are becoming clearer over time, the vast majority of herbs and spices still have rather ill-defined health benefits and yet-to-be-identified chemical "actors" [4].

Given the widespread use of herbs and spices and given their known (and potential) health benefits, there is clearly a need to better understand the consumption patterns of herbs and spices. Population-wide average dietary intake of common spices varies considerably around the world. For instance, Europeans consume an estimated at $0.5 \mathrm{~g} /$ person per day, Australians and New Zealanders consume between $1.3-1.9 \mathrm{~g} /$ day, and residents of Africa consume $1.8 \mathrm{~g} /$ day. Moderate consumers of herbs and spices are found in the Middle East and Eastern Asia with daily consumption of 2.6 and $3.1 \mathrm{~g} /$ person, respectively. The highest consumers of herbs and spices are found in India, South Africa, and Latin America with an average of $4.4 \mathrm{~g} /$ day [23, 24]. In India, turmeric consumption, alone, has been estimated to be $1.5 \mathrm{~g} /$ person per day [25]. While consumption of herbs and spices is generally higher in Southern countries such as India, Mexico, Peru, China, and Thailand, herb and spice intake has been increasing in many developed countries in Northern Europe and North America, due to changing food habits and a growing preference for ethnic or spicy food [26, 27].

While broad estimates of herb and spice consumption are useful, more detailed information of what herbs/ spices and how much of each are being consumed would be much more useful. In this regard, the development and identification of biomarkers of food intake (BFI) for specific herb and spice consumption would help advance the field. In particular, herb-specific or spice-specific BFIs would permit better exposure estimates for much more comprehensive and far more detailed epidemiological studies of the influence of herbs and spices on human health. This review is focused on finding and evaluating specific nutritional biomarkers for a representative set of 25 common herbs and spices used worldwide $[3,10]$.

\section{Methods}

\section{Selection of herbs and spices}

This set of 25 was selected based on reported estimates of consumption volume in North America and Europe as well as the frequency with which these spices and herbs were cited in the literature. The 25 herbs and spices that were examined include anise, basil, black pepper, caraway, Capsicum sp. (chili pepper and paprika), cinnamon, clove, cumin, curcumin, dill, fennel, fenugreek, ginger, lemongrass, marjoram, nutmeg, oregano, parsley, peppermint and spearmint, rosemary, saffron, sage, tarragon, and thyme (Table 1).

\section{Primary literature search}

A systematic BFI review should consist of an extensive literature search (ELS) for each food or food group, which will select from all the putative markers proposed in the available scientific literature only the most promising candidate biomarkers based on their likely specific presence in the food and/or food group [28]. The structure of the present guidelines for conducting an ELS on putative and candidate BFIs is reported elsewhere [29], which follows the methods proposed by the European Food Safety Authority (EFSA) for conducting systematic reviews for food and feed safety assessments [30], as well as the 'Cochrane handbook for systematic review on interventions' [31], with proper modifications for handling BFIs. The PRISMA statement for reporting and discussing the results [32] was also used. Original research papers and reviews were searched in three databases: PubMed, Scopus, and the ISI Web of Knowledge using combinations of the grouped search terms (biomarker* OR marker* OR metabolite* OR biokinetics OR biotransformation) AND (trial OR experiment OR study OR intervention) AND (human* OR men OR women OR patient* OR volunteer* OR participant*) AND (urine OR plasma OR serum OR blood OR excretion) AND (intake OR meal OR diet OR ingestion OR consumption OR eating OR drink* OR administration) as reported in in Additional file 1: Table S1, together with specific keywords related to herbs and spices food group (Additional file 1:Table S2). The specific search terms for specific herbs and spices included both their common and scientific names so as to be as comprehensive as possible. The specific keywords for the herbs and spices of interest were the following: ("anise" OR "Pimpinella anisum" OR "basil" OR "Ocimum basilicum" OR "black pepper" OR "Piper nigrum" OR "caraway" OR "Carum carvi" OR "chili pepper" OR "Capsicum annuum" OR "Capsicum baccatum" OR "Capsicum chinense" OR "Capsicum frutescens" OR "Capsicum pubescens" OR "cinnamon" OR "Cinnamomum" OR "clove" OR "Syzygium aromaticum" OR "cumin" OR "Cuminum cyminum" OR "turmeric" OR "Curcuma longa" OR "dill" OR "Anethum graveolens" OR "fennel" OR "Foeniculum vulgare" OR "fenugreek" OR "Trigonella foenum-graecum" OR "ginger" OR "Zingiber officinale" OR "lemongrass" OR "Cymbopogon" OR "marjoram" OR "Origanum majorana" OR "nutmeg" OR "Myristica fragrans" OR "oregano" OR "Origanum vulgare" OR "parsley" OR "Petroselinum crispum" OR "peppermint" OR "Mentha $x$ piperita" OR "rosemary" OR "Rosmarinus officinalis" OR "saffron" OR "Crocus sativus" OR "sage" OR "Salvia officinalis" OR "spearmint" OR "Mentha spicata" OR "tarragon" OR "Artemisia dracunculus" OR "thyme" OR 
"Thymus vulgaris". To improve the accuracy of the search and to help exclude unrelated papers, several other keywords were used including NOT (Ginger [Author] OR Parsley [Author] OR Sage [Author] OR Dill [Author] OR Pimpinella [Author] OR Basil [Author] OR Artemisia [Author] OR Cumin [Author] OR Thyme [Author]). The default search fields for each of the databases were [All Fields] for PubMed, [Article Title/ Abstract/ Keywords] for Scopus, and [Topic] for ISI Web of Science, respectively. This literature search was conducted between November 2015 and January of 2016, followed by a second validation phase and update in January 2018. The literature search was limited to papers in the English language with no restrictions being applied for the publication dates. Results of this literature search for potential biomarkers of herbs and spices are shown in Table 2.

\section{Exclusion criteria}

Papers were excluded if they investigated the effect on human physiology of the selected herbs and spices, the presence or effect of toxicants, if they referred to unspecific markers or if they were based on in vitro or animal studies.

\section{BFI identification and classification}

A second search step was used to evaluate the apparent specificity of the markers in the list. The remaining list of potential biomarkers was used for a second literature search in the three bibliographic databases used also for the primary search. This was done in order to identify other foods containing the potential biomarkers or their precursors, as well as foods otherwise associated with these compounds. For the second web-based literature search, the "marker name" was used as keyword, together with AND (biomarker* OR marker* OR metabolite $^{*}$ OR biokinetics OR biotransformation). Further filters, such as (urine OR plasma OR serum OR blood OR excretion) AND (intake OR meal OR diet OR ingestion OR consumption OR eating OR drink* OR administration) AND (human* OR men OR women OR patient* OR volunteer* OR participant* OR subject*) were added based on the results obtained. At the end of this selection process, the usefulness, and weakness of each biomarker compounds were evaluated, and the most promising biomarkers were scored to assess their validity as BFIs according to the system reported below.

\section{Marker validation score}

In order to further assess the validity of the biomarker candidates, a set of consensus evaluation criteria, from the FoodBAll consortium was employed [33]. Specifically, the suitability of each biomarker was assessed by answering a set of questions reported elsewhere [33], which reflect the analytical and biological criteria that the proposed biomarkers should fulfill in order to be considered valid. Such questions have been answered for the most promising biomarkers and the results are reported in Table 3. Possible answers were $\mathrm{Y}$ (yes), N (no), or $\mathrm{U}$ (unknown or uncertain). The potential markers were scored for plausibility and uniqueness (question 1); kinetics and dose-response relationship (question 2), kinetics of postprandial response (question 3a) and longer-term kinetics (question 3b). All markers were further evaluated for their robustness in complex diets or a real exposure situation (question 4) and reliability (question 5), which refers to the concordance with other measures of intake for the food or food group in question (such as other existing validated biomarkers or dietary instruments). The analytical aspects of each BFI were investigated through an evaluation of their chemical stability (question 6), their analytical performance (question 7), and reproducibility in different labs (question 8).

\section{Results}

The research papers that identified, described, or evaluated potential biomarkers of intake for the set of 25 herbs and spices were further screened by one or more skilled researchers as described in Fig. 1. The initial PubMed search retrieved 527 matches, the Web of Science search generated 370 matches, and the Scopus search generated 284 matches, resulting in a total of 1181 hits. This number was reduced to 946 after the removal of duplicates. Subsequent screening of the titles and abstracts by the skilled researchers reduced the number of papers to 54. Further evaluation excluded 6 papers and a secondary search identified 1 more paper leading to a total of 49 papers that were included in this review (Fig. 1). Additional papers were identified from the reference lists in these papers and from reviews or book chapters identified through the literature search. This secondary search was used to evaluate the apparent specificity of the marker.

From this total of 49 papers, 18 BFI papers were found for turmeric and curcumin, 6 papers were found for peppermint, 4 papers were found for thyme, 3 papers for ginger, Capsicum and parsley, 2 papers for anise and saffron; and 1 paper each was found for cinnamon, fennel, nutmeg, oregano, marjoram, rosemary and sage, and finally one paper which examined basil, tarragon and fennel (Table 2). No potential BFI papers were found for black pepper, caraway, clove, cumin, dill, fenugreek, lemongrass, and spearmint.

A systematic validation of the candidate intake biomarkers is assessed and presented in Table 3. It should be noted that the search criteria were primarily targeted for BFIs in humans, but, in some cases, a small number of papers in which the BFI study was performed in animals were also investigated. These papers were used to provide supportive information to the studies performed in humans; however, the biomarkers observed only in animal studies were not considered eligible for further BFI validation. Figure 2 
Table 2 List of studies reporting candidate biomarkers for herb and spice intake

\begin{tabular}{|c|c|c|c|c|c|c|}
\hline Dietary factor & Study design & Subjects & $\begin{array}{l}\text { Analytical } \\
\text { method }\end{array}$ & Sample type & $\begin{array}{l}\text { Discriminating } \\
\text { metabolites/candidate } \\
\text { biomarkers }\end{array}$ & $\begin{array}{l}\text { Primary } \\
\text { Ref. }\end{array}$ \\
\hline $\begin{array}{l}\text { Anise (anethole } \\
\text { admn.) }\end{array}$ & $\begin{array}{l}\text { Acute human study. } \\
{\left[\text { methoxy }-{ }^{14} \mathrm{C} \text {-labeled }\right.} \\
\text { compound }\end{array}$ & 5 (males) & $\begin{array}{l}\text { Radiochemical } \\
\left({ }^{14} \mathrm{C} \text { labeled }\right) \\
\text { and HPLC }\end{array}$ & $\begin{array}{l}\text { Urine }(2 \mathrm{~h}-10 \mathrm{~h}, 24 \mathrm{~h} \text {, } \\
\text { and } 48 \mathrm{~h})\end{array}$ & $\begin{array}{l}\text { 4-methoxybenzoic acid, 4- } \\
\text { methoxyhippuric acid, } 3 \\
\text { unknown compounds }\end{array}$ & {$[36]$} \\
\hline \multirow[t]{2}{*}{$\begin{array}{l}\text { Anise-based } \\
\text { alcoholic drink }\end{array}$} & $\begin{array}{l}\text { Dose escalating study } \\
(120 \mathrm{ml}, 200 \mathrm{ml}, 360 \mathrm{ml} \\
\text { "Helenas Ouzo" } \\
\text { (anethole-containing } \\
\text { drink) }\end{array}$ & 1 & $\begin{array}{l}\text { HS-SPME-GC- } \\
\text { MS }\end{array}$ & $\begin{array}{l}\text { Serum }(1,2,4,8 \text {, and } 24 \\
\text { h) }\end{array}$ & Anethole & {$[37]$} \\
\hline & $\begin{array}{l}\text { Observational study: } \\
\text { drivers under the } \\
\text { influence of alcoholic- } \\
\text { containing anethole } \\
\text { drink }\end{array}$ & 50 & & Serum & & \\
\hline $\begin{array}{l}\text { Capsicum sp. } \\
\text { Chili pepper } \\
\text { (capsule) }\end{array}$ & $\begin{array}{l}\text { Acute crossover study ( } 5 \\
\text { g of capsicum extract) }\end{array}$ & 12 (males) & HPLC & Plasma & Capsaicin & [41] \\
\hline $\begin{array}{l}\text { Capsicum sp. } \\
\text { CH-19 sweet } \\
\text { non-pungent } \\
\text { red pepper } \\
\text { (capsule) }\end{array}$ & $\begin{array}{l}\text { Double-blind, } \\
\text { randomized, placebo- } \\
\text { controlled, dose- } \\
\text { escalating ( } 15 \text { or } 30 \mathrm{mg} \\
\text { capsinoids extract) }\end{array}$ & 24 (males) & $\begin{array}{l}\text { LC-MS/MS and } \\
\text { HPLC-UV }\end{array}$ & $\begin{array}{l}\text { Plasma (15 min, } 30 \text { min, } \\
1 \mathrm{~h}, 2 \mathrm{~h}, 4 \mathrm{~h}, 8 \mathrm{~h} \text {, and } \\
24 \mathrm{~h})\end{array}$ & $\begin{array}{l}\text { Capsiate, dihydrocapsiate, } \\
\text { nordihydrocapsiate, } \\
\text { vanillyl alcohol }\end{array}$ & {$[150]$} \\
\hline $\begin{array}{l}\text { Capsicum sp. } \\
\text { Paprika } \\
\text { carotenoids }\end{array}$ & $\begin{array}{l}\text { Case study. } 200 \mathrm{ml} \\
\text { paprika carotenoid } \\
\text { beverage }\end{array}$ & 5 (young, healthy) & $\begin{array}{l}\text { HPLC-UV-VIS } \\
\text { and Q-TOF- } \\
\text { MS/MS }\end{array}$ & $\begin{array}{l}\text { Plasma ( } 0 \text { week, } \\
2 \text { weeks, } 4 \text { weeks), } \\
\text { erythrocytes }\end{array}$ & $\begin{array}{l}\text { B-cryptoxanthin, } \\
\text { cucurbitaxanthin A, } \\
\text { cryptocapsin, lutein, } \\
\text { zeaxanthin, capsanthin, } \\
\text { capsanthone }\end{array}$ & {$[42]$} \\
\hline Cinnamon & $\begin{array}{l}\text { Four-way crossover } \\
\text { study }\end{array}$ & 24 & HPLC MS/MS & Plasma and urine & 7-hydroxycoumarin & {$[48]$} \\
\hline \multirow[t]{2}{*}{$\begin{array}{l}\text { Fennel (fennel } \\
\text { tea) }\end{array}$} & $\begin{array}{l}\text { Single-dose acute study } \\
(500 \mathrm{ml} \text { of fennel tea) }\end{array}$ & 7 & \multirow[t]{2}{*}{$\begin{array}{l}\text { LC-MS/MS and } \\
\text { GC-GC-MS }\end{array}$} & Urine $(1.5,4,8,14,24 \mathrm{~h})$ & \multirow{2}{*}{$\begin{array}{l}\text { Estragole, 1'- } \\
\text { hydroxyestragole, trans- } \\
\text { Anethole, -Allylphenol-G }\end{array}$} & \multirow[t]{2}{*}{ [63] } \\
\hline & $\begin{array}{l}\text { Dose-escalation study } \\
\text { (250, 500, } 1000 \mathrm{ml} \text { fennel } \\
\text { tea). }\end{array}$ & 1 & & Plasma $(0.75,1.5,2,2.5 \mathrm{~h})$ & & \\
\hline $\begin{array}{l}\text { Fennel, basil, } \\
\text { and tarragon }\end{array}$ & $\begin{array}{l}15 \mathrm{~mL} \text { fennel extract; } 15 \\
\mathrm{ml} \text { tarragon extract; } 15 \\
\mathrm{ml} \text { basil brewed }\end{array}$ & NP & $\begin{array}{l}\text { IS-R-DLLME } \\
\text { and HPLC }\end{array}$ & $\begin{array}{l}\text { Plasma ( } 2 \mathrm{~h}, 4 \mathrm{~h} \text {, and } 8 \\
\text { h) and Urine ( } 3 \mathrm{~h}, 6 \mathrm{~h} \text {, } \\
\text { and } 9 \mathrm{~h})\end{array}$ & $\begin{array}{l}\text { Para-anisaldehyde trans- } \\
\text { anethole estragole }\end{array}$ & [38] \\
\hline \multirow[t]{3}{*}{ Ginger (extract) } & $\begin{array}{l}\text { Acute single dose: } 2 \mathrm{~g} \\
\text { ginger extracts }\end{array}$ & 9 (healthy) & LC-MS/MS & $\begin{array}{l}\text { Plasma }(0.25 \text { h, } 0.5 \text { h, } \\
0.75 \text { h, } 1 \text { h, } 2 \text { h, } 4 \text { h, } 6 \text { h, } \\
10 \text { h, } 24 \text { h, } 48 \text { h, and } 72 \\
\text { h) }\end{array}$ & $\begin{array}{l}\text { 10-Gingerol, 6-Shogaol, } \\
\text { 6-Gingerol-G, 8-Gingerol-G } \\
\text { 10-Gingerol-G, 6-Shogaol- } \\
\text { G } \\
\text { 6-Gingerol-S, 8-Gingerol-S, } \\
\text { 10-Gingerol-S, 6-Shogaol-S }\end{array}$ & \multirow[t]{3}{*}{ [70] } \\
\hline & $\begin{array}{l}\text { Multiple dose: } 24 \text {-day } \\
\text { randomized controlled } \\
\text { trial. } 250 \mathrm{mg} \text { ginger } \\
\text { extract }\end{array}$ & 30 (healthy) & & $\begin{array}{l}\text { Plasma (0-24 h) and } \\
\text { colon (biopsy) }\end{array}$ & $\begin{array}{l}\text { 6-Gingerol-G (plasma), 10- } \\
\text { Gingerol-G (plasma), 6- } \\
\text { Gingerol-S (plasma), 10- } \\
\text { Gingerol-G (colon), 10- } \\
\text { Gingerol-S (colon) }\end{array}$ & \\
\hline & $\begin{array}{l}\text { Multiple dose: } 24 \text {-day } \\
\text { randomized controlled } \\
\text { trial. } 250 \mathrm{mg} \text { ginger } \\
\text { extract }\end{array}$ & $\begin{array}{l}20 \text { (high-risk colorectal } \\
\text { cancer) }\end{array}$ & & $\begin{array}{l}\text { Plasma (0-24 h) and } \\
\text { colon (biopsy) }\end{array}$ & $\begin{array}{l}\text { 6-Gingerol-G (plasma), 10- } \\
\text { Gingerol-G (plasma), 6- } \\
\text { Gingerol-S (plasma) 10- } \\
\text { Gingerol-G (colon), 10- } \\
\text { Gingerol-S (colon) }\end{array}$ & \\
\hline Ginger & $\begin{array}{l}\text { Dose escalation study: } \\
100 \mathrm{mg}, 250 \mathrm{mg}, 500 \mathrm{mg}, \\
1 \mathrm{~g}, 1.5 \mathrm{~g}, 2 \mathrm{~g} \text { ginger } \\
\text { extract (capsule) }\end{array}$ & 27 (healthy) & $\begin{array}{l}\text { HPLC-ECD, } \\
\text { HPLC-UV }\end{array}$ & $\begin{array}{l}\text { Plasma (15 min, } 30 \text { min, } \\
\text { and } 45 \text { min, } 1 \text { h, } 2 \mathrm{~h} \text {, } \\
4 \mathrm{~h}, 6 \mathrm{~h}, 10 \mathrm{~h}, 24 \mathrm{~h} \text {, } \\
48 \mathrm{~h} \text {, and } 72 \mathrm{~h} \text { ) }\end{array}$ & $\begin{array}{l}\text { 6-Gingerol-G, 8-Gingerol- } \\
\text { G, 10-Gingerol-G, 6- } \\
\text { Shogaol-G, 6-Gingerol-S, } \\
\text { 10-Gingerol-S }\end{array}$ & [69] \\
\hline $\begin{array}{l}\text { Ginger (ginger } \\
\text { tea) }\end{array}$ & $\begin{array}{l}\text { Acute study. } 2 \times(18 \mathrm{~g} / \\
\text { bag) ginger tea (focused }\end{array}$ & 3 (healthy males) & LC/ESI-MS/MS & $\begin{array}{l}\text { Urine }(0-2 \text { h, 2-4 h, 4- } \\
6 \text { h, 6-9 h, 9-12 h, and }\end{array}$ & $\begin{array}{l}\text { 5-Cys-6S, 5-NAC-6S, 5-Cys- } \\
\text { Gly-6S, 5-Cys-M6, 5-NAC- }\end{array}$ & [71] \\
\hline
\end{tabular}


Table $\mathbf{2}$ List of studies reporting candidate biomarkers for herb and spice intake (Continued)

\begin{tabular}{|c|c|c|c|c|c|c|}
\hline Dietary factor & Study design & Subjects & $\begin{array}{l}\text { Analytical } \\
\text { method }\end{array}$ & Sample type & $\begin{array}{l}\text { Discriminating } \\
\text { metabolites/candidate } \\
\text { biomarkers }\end{array}$ & $\begin{array}{l}\text { Primary } \\
\text { Ref. }\end{array}$ \\
\hline & $\begin{array}{l}\text { on the metabolism of } \\
\text { shogaol) }\end{array}$ & & & $12-24$ h) & $\begin{array}{l}\text { M6, 5-Cys-Gly-M6, 5-Cys- } \\
8 S^{2} \text { 5-Cys-M6', 5-Cys-10S, } \\
\text { 5-Cys-M6" }\end{array}$ & \\
\hline $\begin{array}{l}\text { Marjoram } \\
\text { (extract) }\end{array}$ & $\begin{array}{l}\text { Acute single oral dose } \\
(3.75 \mathrm{~g}) \text { of } O \text {. onites } \\
\text { extract }\end{array}$ & 6 (healthy) & HPLC-CEAD & Urine (24h, 48 h) & $\begin{array}{l}\text { Protocatechuic acid, p- } \\
\text { hydroxybenzoic acid, } \\
\text { caffeic acid, ferulic acid, } \\
\text { syringic acid, vanillic acid, } \\
\text { p-coumaric acid, 3,4- } \\
\text { dihydroxyphenylacetic } \\
\text { acid, } m \text { - } \\
\text { hydroxyphenylacetic acid }\end{array}$ & [97] \\
\hline \multirow[t]{2}{*}{ Nutmeg } & $\begin{array}{l}\text { Acute oral dose in rats } \\
\text { ( } 100 \mathrm{mg} / \mathrm{kg} \text { body mass) } \\
\text { of EL, MY, and SA or a } \\
\text { single } 500 \mathrm{mg} / \mathrm{kg} \text { body } \\
\text { mass of nutmegs }\end{array}$ & $\begin{array}{l}2 \text { rats } \times \text { each } \\
\text { substance and dose }\end{array}$ & GC-MS & Urine (24 h) & \multirow[t]{2}{*}{$\begin{array}{l}\text { O-demethyl elemicin", O- } \\
\text { demethyl dihydroxy ele- } \\
\text { micin }^{*} \text {, demethylenyl myr- } \\
\text { isticin*", dihydroxy } \\
\text { myristicin", demethylenyl } \\
\text { safrole }^{*}\end{array}$} & \multirow[t]{2}{*}[73]{} \\
\hline & $\begin{array}{l}\text { Observational } \\
\text { exploratory toxicological } \\
\text { study: after nutmeg } \\
\text { abuse ( } 5 \text { nutmegs) }\end{array}$ & 1 & & & & \\
\hline $\begin{array}{l}\text { Oregano } \\
\text { (extract) }\end{array}$ & $\begin{array}{l}\text { Oregano extract }(25,75 \text {, } \\
\text { or } 225 \mathrm{mg} / \mathrm{kg}\end{array}$ & 15 mice & HPLC-MS/MS & Plasma and brain tissue & Carvacrol & {$[95]$} \\
\hline Parsley & $\begin{array}{l}\text { Randomized crossover } \\
\text { with two 1-week inter- } \\
\text { vention periods in suc- } \\
\text { cession, supplemented } \\
\text { with parsley } 20 \mathrm{~g} \text { pars- } \\
\text { ley/MJ }\end{array}$ & 14 (healthy) & HPLC-DAD & Urine (24 h) & Apigenin & $\begin{array}{l}{[108,} \\
109]\end{array}$ \\
\hline Parsley & $\begin{array}{l}\text { Acute human study. } \\
(149.45 \pm 35.21 \mathrm{~g} \text { parsley) }\end{array}$ & 11 (healthy) & HPLC-ECD & $\begin{array}{l}\text { Plasma (4-11 h, } 28 \mathrm{~h}) \text {, } \\
\text { urine ( } 24 \mathrm{~h}) \text {, and red } \\
\text { blood cells }\end{array}$ & Apigenin & [102] \\
\hline \multirow{2}{*}{$\begin{array}{l}\text { Peppermint oil } \\
\text { (capsule) }\end{array}$} & \multirow{2}{*}{$\begin{array}{l}\text { Acute pharmacokinetic } \\
\text { study. Intake of } 0.4 \mathrm{ml} \\
\text { peppermint oil in either } \\
\text { colpermin or gelatine } \\
\text { capsules (91-97 mg } \\
\text { capsule) }\end{array}$} & 6 (healthy) & \multirow[t]{2}{*}{ NP } & \multirow[t]{2}{*}{ Urine (24 h) } & \multirow[t]{2}{*}{ Menthol-G } & \multirow[t]{2}{*}{ [117] } \\
\hline & & 6 (ileostomy) & & & & \\
\hline Peppermint oil & $\begin{array}{l}\text { Acute randomized intake } \\
\text { of } 0.6 \mathrm{ml} \text { peppermint oil } \\
\text { in either Colpermin or } \\
\text { Mintec preparations }\end{array}$ & 13 (healthy) & GC-MS & $\begin{array}{l}\text { Urine }(2 \mathrm{~h} \text {-interval for } \\
14 \mathrm{~h}+\text { single overnight } \\
(10 \mathrm{~h})\end{array}$ & Menthol-G & [116] \\
\hline $\begin{array}{l}\text { Peppermint oil } \\
\text { (capsule) }\end{array}$ & $\begin{array}{l}180 \text { mg peppermint oil } \\
\text { enteric-coated capsule } \\
\text { (peroral administration) }\end{array}$ & 4 (males) & GC-FID & $\begin{array}{l}\text { Urine (2-h interval up to } \\
14 \mathrm{~h})\end{array}$ & Menthol-G & [118] \\
\hline $\begin{array}{l}\text { Peppermint oil } \\
\text { (capsule) }\end{array}$ & $\begin{array}{l}\text { Acute ( } 400 \text { mg } \\
\text { peppermint oil in } \\
\text { enteric-coated capsule) } \\
\text { and repeated } 4 \text { weeks } \\
\text { later }\end{array}$ & 5 (healthy) & ${ }^{2} \mathrm{H}-\mathrm{NMR}$ & $\begin{array}{l}\text { Urine ( } 2 h, 4 h, 6 h \text {, and } \\
8 h)\end{array}$ & Menthol-G & [120] \\
\hline \multirow[t]{2}{*}{$\begin{array}{l}\text { Peppermint oil } \\
\text { (capsule) }\end{array}$} & $\begin{array}{l}\text { (1) } 400 \text { mg of enteric- } \\
\text { coated peppermint oil } \\
\text { capsules and } 6 \mathrm{~g} \text { of } 99 \% \\
{\left[\mathrm{U}^{13} \mathrm{C}\right] \text { glucose }}\end{array}$ & 1 (female) & ${ }^{13} \mathrm{C}-\mathrm{NMR}$ & Urine $(2-4 h)$ & ${ }^{13} \mathrm{C}$-menthol-G & [119] \\
\hline & $\begin{array}{l}\text { (2) Primed infusion of } \\
{\left[U^{-13} \mathrm{C}\right] \text { glucose }+400} \\
\text { mg enteric-coated } \\
\text { peppermint oil capsules }\end{array}$ & 4 (severe heart failure) & & Urine (2 h) & & \\
\hline $\begin{array}{l}\text { Peppermint oil } \\
\text { (L-menthol }\end{array}$ & $\begin{array}{l}\text { Escalating-single-dose, } \\
\text { randomized, double- }\end{array}$ & 24 (males) & GC-MS & $\begin{array}{l}\text { Plasma }(5,10,30,60, \\
120 \text {, and } 240 \text { min and } 8,\end{array}$ & $\begin{array}{l}\text { Menthol, menthol-G, M7, } \\
\text { M9, M11, M29 }\end{array}$ & [121] \\
\hline
\end{tabular}


Table $\mathbf{2}$ List of studies reporting candidate biomarkers for herb and spice intake (Continued)

\begin{tabular}{|c|c|c|c|c|c|c|}
\hline Dietary factor & Study design & Subjects & $\begin{array}{l}\text { Analytical } \\
\text { method }\end{array}$ & Sample type & $\begin{array}{l}\text { Discriminating } \\
\text { metabolites/candidate } \\
\text { biomarkers }\end{array}$ & $\begin{array}{l}\text { Primary } \\
\text { Ref. }\end{array}$ \\
\hline \multirow[t]{2}{*}{ preparation) } & \multirow{2}{*}{$\begin{array}{l}\text { blind, placebo-controlled } \\
\text { (menthol preparation, } \\
80-320 \mathrm{mg} \text { ). Intragastric } \\
\text { spraying of peppermint } \\
\text { oil }\end{array}$} & & & $\begin{array}{l}\text { 12, and } 24 \mathrm{~h} \text { after each } \\
\text { dose) }\end{array}$ & & \\
\hline & & & & $\begin{array}{l}\text { Urine (before dosing } \\
(-12-0 \mathrm{~h}) \text { and } 0-4 \mathrm{~h}, 4- \\
8 \mathrm{~h}, 8-12 \mathrm{~h} \text {, and } 12-24 \mathrm{~h} \\
\text { after }\end{array}$ & $\begin{array}{l}\text { Menthol-G, M2, M3-11, } \\
\text { M12, M13-18, M19-21, } \\
\text { M22-28, M29, M30-32. }\end{array}$ & \\
\hline \multirow[t]{2}{*}{$\begin{array}{l}\text { Rosemary } \\
\text { (extract) }\end{array}$} & $\begin{array}{l}\text { Acute, controlled, } \\
\text { randomized study. } \\
\text { Rosemary extract } \\
\text { enriched in carnosic acid } \\
40 \%(w / W)\end{array}$ & 24 Zucker rats & $\begin{array}{l}\text { HPLC/QTOF- } \\
\text { MS and HPLC- } \\
\text { UV }\end{array}$ & Gut, liver, plasma, brain, & \multirow{2}{*}{$\begin{array}{l}\text { Carnosic acid-G, carnosol- } \\
\text { G, rosmanol-G, carnosic } \\
\text { acid } 12 \text { methyl ether, } \\
\text { 5,6,7,10-tetrahydro-7- } \\
\text { hydroxyrosmariquinone, } \\
\text { carnosic glutathione oxi- } \\
\text { dized, carnosol-S, } \\
\text { rosmanol-S, rosmarinic } \\
\text { acid, carnosic cysteine, } \\
\text { carnosic glutathione, } \\
\text { rosmadial-G, rosmanol, } \\
\text { ipirosmanol, epiisorosma- } \\
\text { nol, rosmadial/rosmanol } \\
\text { quinone, rosmanol/epiros- } \\
\text { manol methyl ether, car- } \\
\text { nosol, rosmadial methyl } \\
\text { ether, epirosmanol ethyl } \\
\text { ether, epiisorosmanol me- } \\
\text { thyl ether, carnosol me- } \\
\text { thyl ether, carnosic acid. }\end{array}$} & \multirow{2}{*}{ [100] } \\
\hline & $\begin{array}{l}\text { Subchronic, controlled, } \\
\text { randomized study } \\
\text { Rosemary extract } \\
\text { enriched in carnosic acid } \\
40 \%(w / W) \text { ( } 64 \text { days) }\end{array}$ & & & & & \\
\hline Saffron (tea) & $\begin{array}{l}\text { Single-dose acute study. } \\
200 \mathrm{mg} \text { saffron in } 150 \mathrm{ml} \\
\text { water (saffron tea) }\end{array}$ & 4 (healthy) & SPE-HPLC-DAD & $\begin{array}{l}\text { Plasma (0 h, } 2 \text { h, and } 24 \\
\text { h) }\end{array}$ & cis-Crocetin, trans-Crocetin & [126] \\
\hline $\begin{array}{l}\text { Saffron (purified } \\
\text { crocetin) }\end{array}$ & $\begin{array}{l}\text { Open-label, single dose } \\
\text { escalation of crocetin } \\
(7.5,15 \text { and } 22.5 \mathrm{mg})\end{array}$ & 10 (healthy) & HPLC & $\begin{array}{l}\text { Plasma }(1,2 \mathrm{~h}, 4 \mathrm{~h}, 6 \mathrm{~h}, \\
8 \mathrm{~h}, 10 \mathrm{~h} \text {, and } 24 \mathrm{~h})\end{array}$ & Crocetin & [127] \\
\hline Sage (tea) & $\begin{array}{l}\text { Acute human study } \\
\text { (1.02 mg 1,8-cineole) in } \\
\text { sage tea }\end{array}$ & 1 (female) & $\begin{array}{l}\text { SPME-GC-MS } \\
\text { and LC-MS/MS }\end{array}$ & $\begin{array}{l}\text { Plasma (0.75 h, } 1.7 \text { h, } \\
3.25 \text { h, } 6.75 \text { h, and } 24 \text { h) } \\
\text { and urine (2 h, } 5 \text { h, } 7 \text { h, } \\
10 \text { h, } 17 \text { h, } 21 \text { h, } 28 \text { h, } \\
32 \text { h, } 35 \text { h, } 44 \text { h, } 50 \text { h, } \\
53 \text { h, } 60 \text { h, and } 69 \text { h) }\end{array}$ & $\begin{array}{l}\text { 1,8-cineole, 2-hydroxy-1,8- } \\
\text { cineole, 3-hydroxy-1,8-cin- } \\
\text { eole, 7-hydroxy-1,8-cine- } \\
\text { ole, 9-hydroxy-1,8-cineole. }\end{array}$ & [130] \\
\hline \multirow[t]{2}{*}{ Thyme (tablet) } & \multirow[t]{2}{*}{$\begin{array}{l}\text { Acute study. A single } \\
\text { dose of a Bronchipret } \\
\text { TP (tablet equivalent to } \\
1.08 \text { mg thymol) }\end{array}$} & \multirow[t]{2}{*}{12} & \multirow[t]{2}{*}{$\begin{array}{l}\text { HS-SPME-GC- } \\
\text { MS and LC- } \\
\text { MS/MS }\end{array}$} & $\begin{array}{l}\text { Plasma (0.25 h, } 0.5 \text { h, } \\
0.75 \text { h, } 1 \text { h, } 1.5 \text { h, } 2 \text { h, } \\
2.5 \text { h, } 3 \text { h, } 3.5 \text { h, } 4 \text { h, } 5 \text { h, } \\
6 \text { h, } 7 \text { h, } 8 \text { h, } 9 \text { h, } 10 \text { h, } \\
11 \text { h, } 12 \text { h, } 14 \text { h, } 24 \text { h, } \\
31 \text { h, } 38 \text { h, } 48 \text { h, } 55 \text { h, } \\
62 \text { h, and } 72 \text { h) }\end{array}$ & Thymol-S & \multirow[t]{2}{*}{ [151] } \\
\hline & & & & $\begin{array}{l}\text { Urine ( } 0 \text { to } 3 \mathrm{~h}, 3 \text { to } 6 \mathrm{~h} \text {, } \\
6 \text { to } 9 \mathrm{~h}, 9 \text { to } 14 \mathrm{~h}, 14 \\
\text { to } 24 \mathrm{~h}, 24 \text { to } 31 \mathrm{~h}, 38 \\
\text { to } 48 \mathrm{~h}, 48 \text { to } 55,55 \text { to } \\
62 \mathrm{~h} \text {, and } 62 \text { to } 72 \mathrm{~h} \text { ) }\end{array}$ & Thymol-G, thymol-S & \\
\hline Thyme & $\begin{array}{l}\text { Acute intake of } 1.5 \mathrm{~g} \text { of } \\
\text { thyme extract }\end{array}$ & 12 Wistar rats & $\begin{array}{l}\mu S P E-U P L C- \\
M S / M S\end{array}$ & Plasma & $\begin{array}{l}\text { Thymol-S, thymol-G, } \\
\text { luteolin-S, luteolin-G, } \\
\text { hydroxyphenylpropionic } \\
\text { acid-S, coumaric acid-S, } \\
\text { caffeic acid-S, ferulic acid- } \\
\text { S, ferulic acid-G, hydroxy- } \\
\text { benzoic acid, and dihy- } \\
\text { drophenylpropionic acid-S }\end{array}$ & [98] \\
\hline $\begin{array}{l}\text { Thyme (olive oil } \\
\text { enriched with } \\
\text { thyme }\end{array}$ & $\begin{array}{l}\text { Randomized, double- } \\
\text { blind, controlled, cross- } \\
\text { over trial. Administration }\end{array}$ & $\begin{array}{l}33 \\
\text { (hypercholesterolemic) }\end{array}$ & $\begin{array}{l}\text { MSPE-UPLC-ESI- } \\
\text { MS/MS }\end{array}$ & Plasma & $\begin{array}{l}\text { Thymol-S, } \\
\text { hydroxyphenylpropionic } \\
\text { acid-S, caffeic acid-S }\end{array}$ & [99] \\
\hline
\end{tabular}


Table $\mathbf{2}$ List of studies reporting candidate biomarkers for herb and spice intake (Continued)

\begin{tabular}{|c|c|c|c|c|}
\hline Dietary factor & Study design & Subjects & $\begin{array}{l}\text { Analytical } \\
\text { method }\end{array}$ & Sample type \\
\hline polyphenols) & $\begin{array}{l}\text { of } 25 \mathrm{ml} / \text { day }(\mathrm{VOO}) / \mathrm{NOO} \\
+\mathrm{PC} \mathrm{NOO}+\mathrm{PC}+\mathrm{PC} \text { of } \\
\text { thyme }\end{array}$ & & & Urine $(24 \mathrm{~h})$ \\
\hline $\begin{array}{l}\text { Thyme (olive oil } \\
\text { enriched with } \\
\text { thyme) }\end{array}$ & $\begin{array}{l}\text { (1) In vitro colonic } \\
\text { fermentation ( } 0 \text { to } 48 \mathrm{~h} \text { ) }\end{array}$ & 3 (healthy) & $\begin{array}{l}\text { UPLC-ESI-MS/ } \\
\text { MS and GC- } \\
\text { FID }\end{array}$ & $\begin{array}{l}\text { Feces (in vitro } \\
\text { fermentation) }\end{array}$ \\
\hline
\end{tabular}

(2) Human intervention study: $25 \mathrm{ml} /$ day of a thyme phenol-enriched olive oil for 3 weeks

\begin{tabular}{|c|c|}
\hline $\begin{array}{l}\text { Turmeric } \\
\text { (curcuminoids in } \\
\text { capsule) }\end{array}$ & $\begin{array}{l}\text { Randomized double } \\
\text { blind placebo (1 } \mathrm{g} / \text { day, } \\
4 \mathrm{~g} / \text { day, placebo), } \\
6 \text { months }\end{array}$ \\
\hline $\begin{array}{l}\text { Turmeric } \\
\text { (curcuminoids in } \\
\text { capsule) }\end{array}$ & Acute study \\
\hline $\begin{array}{l}\text { Turmeric } \\
\text { (curcuminoidsin } \\
\text { nanoemulsion) }\end{array}$ & $\begin{array}{l}\text { Acute study ( } 2 \mathrm{~g} \\
\text { nanoemulsion } \\
\text { curcuminoids) }\end{array}$ \\
\hline $\begin{array}{l}\text { Turmeric } \\
\text { (curcuminoids in } \\
\text { capsule) }\end{array}$ & $\begin{array}{l}\text { Nonrandomized, open- } \\
\text { label, phase II trial (start- } \\
\text { ing dose } 8 \mathrm{~g} \text { curcumi- } \\
\text { noids) } 8 \text { weeks }\end{array}$ \\
\hline $\begin{array}{l}\text { Turmeric } \\
\text { (curcuminoids in }\end{array}$ & $\begin{array}{l}\text { Dose escalation. } 450- \\
3600 \text { mg/day } 1 \text { week }\end{array}$ \\
\hline
\end{tabular}

10

31 (elderly)

2 (healthy)

2 (healthy)

LC-MS/MS

Plasma

25 (pancreatic cancer) LC-MS

Plasma ( 1 h, 2 h, 6 h $24 h, 48$ h, 72 h, day 8 and after 4 weeks

12 (hepatic metastasis HPLC-UV, LCfrom colorectal month)

Plasma and liver tissue

Discriminating
metabolites/candidate
biomarkers
Thymol-S, Thymol-G,
hydroxyphenylpropionic
acid-S, $p$-cymene-diol-G,
caffeic acid-S

caffeic acid-S

Thymol, carvacrol, 2-(3',4'dihydroxyphenyl) acetic acid, 2-(4'-hydroxyphenyl) acetic acid, phenylacetic acid, 3-(4'-hydroxyphenyl) propionic acid,

phenylpropionic acid.

2-(3',4'-dihydroxyphenyl) acetic acid, 2-(4'hydroxyphenyl) acetic acid, Phenylacetic acid, 3(4'-hydroxyphenyl) propionic acid, phenylpropionic acid

Caffeic acid, $p$-coumaric acid, 3-(3', 4'-

dihydroxyphenyl) propionic acid; hydroxyphenylpropionic acid; phenylpropionic acid, 2-(3',4'-

dihydroxyphenyl) acetic acid; 2-(4'-hydroxyphenyl) acetic acid; phenylacetic acid

3-(3', 4'-dihydroxyphenyl) propionic acid; hydroxyphenylpropionic acid; phenylpropionic acid, 2-(3',4'dihydroxyphenyl) acetic acid, 2-(4'-hydroxyphenyl) acetic acid; phenylacetic acid

Feces (in vivo, (0-3wk) Carvacrol, 2-(4hydroxyphenyl) acetic acid, 3-(3'-4'dihydroxyphenyl) propionic acid, hydroxyphenylpropionic acid, phenylpropionic acid

Plasma (2-2.5 h after 1 Curcumin, DMC BDMC, THC, ferulic acid, vanillic acid

COG

Curcumin, COG, COS, $\mathrm{DMC}, \mathrm{BDMC}$, and THC

COG and COS

Hexahydrocurcumin (liver),

Prim

[96]

6]

.


Table $\mathbf{2}$ List of studies reporting candidate biomarkers for herb and spice intake (Continued)

\begin{tabular}{|c|c|c|c|c|c|c|}
\hline Dietary factor & Study design & Subjects & $\begin{array}{l}\text { Analytical } \\
\text { method }\end{array}$ & Sample type & $\begin{array}{l}\text { Discriminating } \\
\text { metabolites/candidate } \\
\text { biomarkers }\end{array}$ & $\begin{array}{l}\text { Primary } \\
\text { Ref. }\end{array}$ \\
\hline capsule) & & cancer) & & & $\begin{array}{l}\text { hexahydrocurcuminol } \\
\text { (liver), curcumin (plasma), } \\
\text { COG (plasma), COS } \\
\text { (plasma). }\end{array}$ & \\
\hline $\begin{array}{l}\text { Turmeric } \\
\text { (curcuminoids in } \\
\text { capsule) }\end{array}$ & Acute study. & $\begin{array}{l}12 \text { (colorectal } \\
\text { carcinoma) }\end{array}$ & $\begin{array}{l}\text { HPLC-UV } \\
\text { HPLC-MS }\end{array}$ & $\begin{array}{l}\text { Plasma and colorectal } \\
\text { tissue }\end{array}$ & $\begin{array}{l}\text { Curcumin (plasma and } \\
\text { colorectal tissue), COG } \\
\text { and COS (colorectal } \\
\text { tissue) }\end{array}$ & {$[6]$} \\
\hline $\begin{array}{l}\text { Turmeric } \\
\text { (curcuminoids- } \\
\text { different } \\
\text { administration } \\
\text { types) }\end{array}$ & $\begin{array}{l}\text { Randomized double } \\
\text { blind crossover study } \\
\text { with formulated (CP, } \\
\text { CTR, CHC) and } \\
\text { unformulated (CS) } \\
\text { curcumin }\end{array}$ & 12 (healthy) & LC-MS/MS & $\begin{array}{l}\text { Plasma }(1 \mathrm{~h}, 2 \mathrm{~h}, 3 \mathrm{~h}, \\
4 \mathrm{~h}, 5 \mathrm{~h}, 6 \mathrm{~h}, 8 \mathrm{~h} \text {, and } \\
12 \mathrm{~h})\end{array}$ & $\begin{array}{l}\text { Curcumin, DMC, BDMC, } \\
\text { THC }\end{array}$ & [138] \\
\hline $\begin{array}{l}\text { Turmeric } \\
\text { (curcuminoids in } \\
\text { capsule) }\end{array}$ & $\begin{array}{l}\text { 14-day intervention } \\
\text { (2.35 g capsule) }\end{array}$ & 24 (colorectal cancer) & $\begin{array}{l}\text { UPLC-UV, LC- } \\
\mathrm{MS} / \mathrm{MS}\end{array}$ & $\begin{array}{l}\text { Plasma, urine and colon } \\
\text { tissue }\end{array}$ & $\begin{array}{l}\text { Curcumin, BDMC, DMC, } \\
\text { BDMC-S, DMC-S, COS, } \\
\text { COG, BDMC-G, DMC-G }\end{array}$ & {$[147]$} \\
\hline $\begin{array}{l}\text { Turmeric } \\
\text { (Theracurmin }^{\oplus)}\end{array}$ & $\begin{array}{l}\text { Acute dose escalation } \\
150 \mathrm{mg} \text { and } 210 \mathrm{mg}\end{array}$ & 6 (healthy) & LC-MS/MS & $\begin{array}{l}\text { Plasma (0 h, } 1 \text { h, 2, } 4 \text { h, } \\
6,24 h)\end{array}$ & Curcumin & [152] \\
\hline $\begin{array}{l}\text { Turmeric } \\
\text { (Theracurmin }^{\oplus} \text { ) }\end{array}$ & $\begin{array}{l}\text { Multi-week dose } \\
\text { escalation }\end{array}$ & $\begin{array}{l}16 \text { (pancreatic or } \\
\text { biliary tract cancer) }\end{array}$ & LC-MS/MS & Plasma (2 h) & Curcumin & [153] \\
\hline \multirow{2}{*}{$\begin{array}{l}\text { Turmeric } \\
\text { (turmeric fresh } \\
\text { derived } \\
\text { curcuminoids vs. } \\
\text { std. curcumin) }\end{array}$} & $\begin{array}{l}\text { Multi-week double } \\
\text { crossover study. } 250 \mathrm{mg} / \\
\text { kg body weight }\end{array}$ & 18 (mice) & $\begin{array}{l}\text { LC-DAD-ESI- } \\
\text { MS/MS }\end{array}$ & $\begin{array}{l}\text { Plasma (0 h, 0.5 , } 1 \text { h, } \\
3 \mathrm{~h}, 5 \mathrm{~h}, 8 \mathrm{~h}, 12 \mathrm{~h})\end{array}$ & Curcumin, DMC, BMDC & [149] \\
\hline & $\begin{array}{l}\text { Acute, single-blind cross- } \\
\text { over study, } 100 \text { mg, } 250 \\
\text { mg, } 1000 \text { mg }\end{array}$ & 15 (healthy) & & & & \\
\hline \multirow[t]{2}{*}{$\begin{array}{l}\text { Turmeric (C3 } \\
\text { complex) }\end{array}$} & $\begin{array}{l}\text { Acute study. } 1 \text { single } \\
\text { dose }(4 \mathrm{~g})\end{array}$ & 8 (healthy) & HPLC & Serum & Curcumin & [154] \\
\hline & $\begin{array}{l}\text { 3-4-week intervention } \\
\text { study. ( } \mathrm{g} / \text { day) }\end{array}$ & 15 (with HNSCC) & & & & \\
\hline $\begin{array}{l}\text { Turmeric } \\
\text { (curcuma } \\
\text { extract capsule) }\end{array}$ & $\begin{array}{l}\text { Dose escalation: } 440 \\
\text { mg- } 2200 \text { mg/day. } \\
4 \text { months }\end{array}$ & 15 (colorectal cancer) & HPLC-UV & Blood, urine, feces & $\begin{array}{l}\text { COS (only detected in } \\
\text { feces) }\end{array}$ & [25] \\
\hline $\begin{array}{l}\text { Turmeric (C3 } \\
\text { complex) }\end{array}$ & $\begin{array}{l}\text { Dose escalation study. } 4- \\
\text { month intervention ( } 450 \text {, } \\
900,1800,3600 \mathrm{mg}) . \\
4 \text { months }\end{array}$ & $\begin{array}{l}15 \text { (colorectal } \\
\text { adenocarcinoma } \\
\text { patients) }\end{array}$ & LC-MS & Plasma, urine, feces & $\begin{array}{l}\text { Curcumin (plasma, urine, } \\
\text { feces), COG (plasma, } \\
\text { urine), DMC (plasma, } \\
\text { urine), BDMC (plasma), } \\
\text { DMC-G (plasma, urine), } \\
\text { DMC-S (plasma) COS } \\
\text { (plasma, urine, and feces). }\end{array}$ & {$[145]$} \\
\hline $\begin{array}{l}\text { Turmeric (C3 } \\
\text { complex, } 10 \text { or } \\
12 \mathrm{~g})\end{array}$ & Acute study & 12 (healthy) & HPLC & Plasma & COG and COS & [139] \\
\hline \multirow[t]{2}{*}{ Turmeric } & \multirow[t]{2}{*}{$\begin{array}{l}\text { 3-month intervention } \\
\text { with different doses } \\
\text { each group (500, 1000, } \\
2000,4000,8000 \mathrm{mg} / \\
\text { day) }\end{array}$} & 25 cancer & HPLC-UV & $\begin{array}{l}\text { Serum }(0 h, 0.5 h, 1 h \text {, } \\
1.5 h, 2 h, 2.5 h, 3 \text { h, } 4 \text { h, } \\
6 h, 8 h, 12 h, 14 h \text {, and } \\
24 h)\end{array}$ & Curcumin (only in serum) & [144] \\
\hline & & & & $\begin{array}{l}\text { Urine }(0-2 h, 2-4 h, 4- \\
8 \text { h, and } 8-24 h)\end{array}$ & & \\
\hline $\begin{array}{l}\text { Turmeric } \\
\text { (Theracurmin }^{\oplus} \text { ) }\end{array}$ & $\begin{array}{l}\text { Acute study. Curcumin } \\
\text { in powder and } \\
\text { Theracurmin in liquid } \\
(30 \mathrm{mg}) \text {. }\end{array}$ & $\begin{array}{l}12 \text { Sprague-Dawley } \\
\text { rats and } 14 \text { humans }\end{array}$ & LC-MS/MS & Plasma & $\begin{array}{l}\text { Theracurmin and } \\
\text { curcumin }\end{array}$ & [148] \\
\hline $\begin{array}{l}\text { Turmeric } \\
\text { (curcumin) }\end{array}$ & $\begin{array}{l}\text { Dose escalation study. } \\
\text { C3 complex adm. }\end{array}$ & 24 & HPLC & Plasma and serum & $\begin{array}{l}\text { Curcumin (just in serum } \\
\text { at } 10000 \text { and } 12,000 \mathrm{mg} \text { ) }\end{array}$ & [146] \\
\hline
\end{tabular}


Table $\mathbf{2}$ List of studies reporting candidate biomarkers for herb and spice intake (Continued)

\begin{tabular}{|c|c|c|c|c|c|c|}
\hline Dietary factor & Study design & Subjects & $\begin{array}{l}\text { Analytical } \\
\text { method }\end{array}$ & Sample type & $\begin{array}{l}\text { Discriminating } \\
\text { metabolites/candidate } \\
\text { biomarkers }\end{array}$ & $\begin{array}{l}\text { Primary } \\
\text { Ref. }\end{array}$ \\
\hline & $\begin{array}{l}2000 \mathrm{mg}, 4000 \\
6000 \mathrm{mg}, 8000 \\
10,000 \mathrm{mg}, \mathrm{an} \\
\mathrm{mg})\end{array}$ & & & & & \\
\hline
\end{tabular}

Adm, administration; BDMC, bisdemethoxycurcumin, $C E A D$, coulometric electrode array detector; $C H C$, combination of hydrophilic carrier, cellulosic derivatives, and natural antioxidants; ${ }^{13} \mathrm{C}-\mathrm{NMR}$, carbon nuclear magnetic resonance; $C O G$, curcumin-O-glucoronide. COS, curcumin-O-sulfate; $C P$, curcumin phytosome formulation; $C S$, standardized curcumin mixture; CTR formulation with volatile oils of turmeric rhizome, Cys, cysteinyl; DAD, diode array detector; DMC, demethoxycurcumin, $E C D$, electrochemical detection. $E L$, elemicin; $E S I$, electrospray ionization; FID, flame ionization detector; $-G$, glucuronide; Gly, glycinyl; $H-N M R$, proton nuclear magnetic resonance; HPLC, high-performance liquid chromatography; HNSCC head and neck squamous cell carcinomas; HS-SPME, headspace solid-phase microextraction; IS-R-DLLME, in-syringe reversed dispersive liquid-liquid microextraction; $L C$, liquid chromatography; months, months; $M J$, megajoules; $M Y$, myristicin, M6, 1-(4'-hydroxy-3'-methoxyphenyl)-4-decen-3-ol; M6', 1-(4'-hydroxy-3'-methoxyphenyl)-4-dodecen-3-ol; M6", 1-(4'-hydroxy-3'-methoxyphenyl)-4tetradecen-3-ol; $M 29$, menthol sulfoconjugate; $M 7,9$, 11, hydroxyl menthol glucuronide. M3-11, hydroxyl menthol glucuronide; M19-21, dihydroxyl menthol glucuronide. M2, aldehyde-menthol glucuronide; M13-18, carboxylate-menthol or aldehyde-hydroxyl menthol glucuronide; M29-32, sulfate conjugates; M12, dialdehydementhol glucuronide (M12); MS mass spectrometry; NAC, N-acetylcysteinyl; NP, not provided; $P C$, phenolic compounds; $Q$-TOF, quadrupole time-offlight; $S$, sulfate; SA, safrole; THC, tetrahydrocurcumin; UPLC, ultra-high performance liquid chromatography; UV ultraviolet; VIS, visible; VOO, virgin olive oil. weeks, week; $w / w$, weight per weight; $\mu S P E$, microelution solid-phase extraction; 6S, 6-shogaol; 8S, 8-shogaol; 10S, 10-shogaol

*In the study performed in rats, there were other metabolites also identified but not found in the human sample analyzed so they were not considered in this table

summarizes many of the findings of this review. It includes the most representative or strongly validated metabolites identified for each herb and spice, the molecule formula, the HMDB ID, and/or Phytohub code (if exists) and the biofluid or tissue in which each BFI has been found.

\section{Anise}

Anise is a seed spice derived from a flowering plant belonging to the family Apiaceae, which is native to the Eastern Mediterranean region and Southwest Asia. The distinctive licorice flavor and aroma from anise comes from anethole. Anethole is a phenylpropene derivative found in anise (Pimpinella anisum) and fennel (Foeniculum vulgare). Anethole occurs naturally in high concentrations in volatile oils such as anise oil (80-90\%), star anise oil (over 90\%), and fennel oil (80\%) [34]. Anethole exists in both a cis and a trans isomer with the trans isomer being more abundant. It is the main component of the anise essential oil (80-90\%), with minor components including para-anisaldehyde, estragole, and pseudo isoeugenyl-2-methylbutyrates, among others [35]. In Mediterranean countries, the popularity of alcoholic and non-alcoholic anise-flavored beverages has led to a much greater consumption of trans-anethole [36]. Anethole is also used in medicines as an expectorant, an antitussive and an antispasmodic for treating gastrointestinal tract illnesses. As a result, anise is found in a number of pharmaceutical products.

Just two papers have reported potential anise intake biomarkers. The most complete study was conducted in 1988 [37], where Caldwell and co-workers performed an acute human study administrating trans-anethole (using a synthesized radio-labeled ${ }^{14} \mathrm{C}$ compound). The major routes of elimination of ${ }^{14} \mathrm{C}$ were in the urine (54-69\% of the administered dose) and as exhaled ${ }^{14} \mathrm{CO}_{2} \quad(13-17 \%)$. The principal metabolite $\left(>90 \%\right.$ of urinary $\left.{ }^{14} \mathrm{C}\right)$ was 4-methoxyhippuric acid (also known as anisic acid), accompanied by much smaller amounts of 4-methoxybenzoic acid (or also known as $p$-anisic acid, an oxidation product of anethole) and up to three other unknown compounds. As the authors stated, metabolism of anethole in man was unaffected by changes in dose size, and is dominated by the $\omega$-oxidation pathway ultimately leading to 4-methoxyhippuric acid, and by oxidative O-demethylation, leading to the exhalation of ${ }^{14} \mathrm{CO}_{2}$ [36].

The only other research article to look into anise BFIs was an observational study that measured the content of anethole in the blood after the intake of alcoholic anise-based beverages [37]. This study rigorously monitored a single individual, wherein the subject consumed the alcoholic drink ouzo over three different days under controlled conditions. In addition to this controlled single-participant study, the authors also looked at the blood collected from 50 motor vehicle drivers who claimed to have consumed drinks containing anethole (ouzo, raki and the German aniseed liqueur "Küstennebel"). The anethole concentrations detected for the tested volunteer showed rapid resorption of anethole as well as rapid elimination. Anethole concentrations above the detection level of $3.6 \mathrm{ng} / \mathrm{ml}$ serum were detected in the selected volunteer for $3 \mathrm{~h}$ after ceasing consumption of $120 \mathrm{ml}$ of Helenas ouzo and for $3 \mathrm{~h}$ after ceasing consumption of $200 \mathrm{ml}$ of regular ouzo, and for $7 \mathrm{~h}$ after ceasing consumption of $360 \mathrm{ml}$ of regular ouzo. For the 50 motor vehicle drivers, 10 out of 50 serum samples had anethole concentrations of between 5.4 and $17.6 \mathrm{ng} /$ $\mathrm{ml}$. Of these, eight corresponded to confirmed cases of ouzo consumption, one of raki consumption and one of German aniseed liqueur "Kustennebel" 
Table 3 Summary of the selected candidate BFls of herbs and spices and their assessment relative to the nine validation criteria/ questions described in [33]

\begin{tabular}{|c|c|c|c|c|c|c|c|c|c|c|c|}
\hline \multirow{2}{*}{$\begin{array}{l}\text { Food item } \\
\text { Anise }\end{array}$} & \multirow{2}{*}{$\begin{array}{l}\text { Metabolites } \\
\text { Anethole }\end{array}$} & \multirow{2}{*}{$\begin{array}{l}\text { Biofluid locations } \\
\text { Serum }\end{array}$} & \multicolumn{9}{|c|}{$\begin{array}{l}\text { Questions }^{*} \\
123 \text { 3a 3b } 45678\end{array}$} \\
\hline & & & $\mathrm{N}$ & Y & $\mathrm{Y}$ & $U$ & $\mathrm{U}$ & $Y$ & $u$ & Y & $\bar{N}$ \\
\hline & 4-methoxyhippuric acid' & Urine & $\mathrm{N}$ & Y & Y & $u$ & U & $u$ & Y & N & $\mathrm{N}$ \\
\hline & 4-methoxybenzoic acid ${ }^{1}$ & Urine & N & Y & Y & $U$ & $U$ & $u$ & Y & N & N \\
\hline \multirow[t]{6}{*}{ Basil } & Estragole & Plasma & $\mathrm{N}$ & Y & Y & $U$ & $\mathrm{U}$ & N & U & Y & N \\
\hline & Estragole & Urine & $\mathrm{N}$ & Y & Y & $\mathrm{U}$ & $\mathrm{U}$ & N & U & Y & N \\
\hline & trans-anethole & Plasma & $\mathrm{N}$ & Y & Y & $U$ & $\mathrm{U}$ & Y & U & Y & N \\
\hline & trans-anethole & Urine & $\mathrm{N}$ & Y & Y & $U$ & $U$ & Y & U & Y & N \\
\hline & para-Anisaldehyde & Plasma & $\mathrm{N}$ & Y & Y & $U$ & $U$ & N & U & Y & N \\
\hline & para-Anisaldehyde & Urine & $\mathrm{N}$ & Y & Y & $U$ & $u$ & N & U & Y & N \\
\hline Capsicum (chili pepper) & Capsaicin & Plasma & Y & Y & Y & U & $\mathrm{U}$ & $\mathrm{u}$ & U & U & N \\
\hline \multirow[t]{4}{*}{ Capsicum (CH-19 sweet non-pungent red pepper) } & Capsiate & Plasma & Y & $U$ & $\mathrm{~N}$ & $U$ & $\mathrm{U}$ & $u$ & $u$ & $\mathrm{~N}$ & N \\
\hline & Dihydrocapsiate & & Y & U & $\mathrm{N}$ & $U$ & U & $u$ & $u$ & N & N \\
\hline & Nordihydrocapsiate & & Y & $U$ & $\mathrm{~N}$ & $U$ & 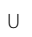 & $u$ & $u$ & N & N \\
\hline & Vanillyl alcohol & & $\mathrm{N}$ & U & $\mathrm{N}$ & $U$ & $\mathrm{U}$ & $u$ & U & $\mathrm{N}$ & N \\
\hline \multirow[t]{7}{*}{ Capsicum (paprika carotenoids) } & $\beta$-Cryptoxanthin & Plasma and erythrocy-tes & $\mathrm{N}$ & Y & N & Y & U & N & U & $\mathrm{N}$ & N \\
\hline & Cucurbitaxanthin A & & Y & Y & $\mathrm{N}$ & Y & U & $u$ & $u$ & N & N \\
\hline & Lutein & & $\mathrm{N}$ & Y & $\mathrm{N}$ & Y & $\mathrm{U}$ & N & U & N & N \\
\hline & Zeaxanthin & & $\mathrm{N}$ & Y & $\mathrm{N}$ & Y & $U$ & N & U & N & N \\
\hline & Capsanthin & & Y & Y & $\mathrm{N}$ & Y & U & $u$ & $u$ & N & N \\
\hline & Capsanthone & & Y & Y & $\mathrm{N}$ & Y & $U$ & U & U & $\mathrm{N}$ & N \\
\hline & Cryptocapsin & & Y & Y & $\mathrm{N}$ & Y & U & U & U & $\mathrm{N}$ & N \\
\hline \multirow[t]{2}{*}{ Cinnamon } & 7-hydroxycoumarin & Plasma & Y & Y & Y & $u$ & Y & U & Y & Y & N \\
\hline & 7-hydroxycoumarin & Urine & Y & Y & Y & $U$ & Y & U & Y & Y & N \\
\hline \multirow[t]{11}{*}{ Fennel } & trans-Anethole & Plasma & $\mathrm{N}$ & Y & Y & $U$ & $\mathrm{U}$ & Y & U & Y & N \\
\hline & trans-Anethole & Urine & $\mathrm{N}$ & Y & Y & $u$ & u & Y & $u$ & Y & N \\
\hline & 1'-hydroxyestragole-G & Plasma & $\mathrm{N}$ & Y & N & $U$ & U & $u$ & U & Y & N \\
\hline & 1'-hydroxyestragole & Urine & $\mathrm{N}$ & Y & N & $U$ & $U$ & U & Y & Y & N \\
\hline & 1'-hydroxyestragole-G & Urine & $\mathrm{N}$ & N & N & $u$ & U & U & u & Y & N \\
\hline & p-Allylphenol-G & Plasma & $\mathrm{N}$ & N & N & $U$ & $\mathrm{U}$ & U & U & Y & N \\
\hline & p-Allylphenol-G & Urine & $N$ & $\mathrm{~N}$ & N & $U$ & $\mathrm{U}$ & U & U & Y & N \\
\hline & Estragole & Plasma & $N$ & Y & Y & $u$ & U & N & u & Y & N \\
\hline & Estragole & Urine & $\mathrm{N}$ & Y & Y & $U$ & $U$ & N & U & Y & N \\
\hline & para-Anisaldehyde & Plasma & $\mathrm{N}$ & Y & Y & $U$ & U & Y & U & Y & N \\
\hline & para-Anisaldehyde & Urine & $\mathrm{N}$ & Y & Y & $U$ & $U$ & Y & U & Y & N \\
\hline \multirow[t]{10}{*}{ Ginger } & 10-Gingerol & Plasma & Y & Y & Y & $N$ & U & U & Y & Y & N \\
\hline & 6-Shogaol & & Y & Y & Y & $\mathrm{N}$ & $U$ & U & Y & Y & N \\
\hline & 6-Gingerol -G & & Y & Y & Y & $\mathrm{N}$ & $\mathrm{U}$ & U & Y & Y & N \\
\hline & 8-Gingerol-G & & Y & Y & Y & $\mathrm{N}$ & U & U & Y & Y & N \\
\hline & 10-Gingerol-G & & Y & Y & Y & $\mathrm{N}$ & U & U & Y & Y & N \\
\hline & 6-Shogaol-G & & Y & Y & Y & $\mathrm{N}$ & $\mathrm{U}$ & $\mathrm{u}$ & Y & Y & N \\
\hline & 6-Gingerol-S & & Y & Y & Y & N & $u$ & $u$ & Y & Y & N \\
\hline & 8-Gingerol-5 & & Y & Y & Y & N & U & U & Y & Y & N \\
\hline & 10-Gingerol-S & & Y & Y & Y & $\mathrm{N}$ & $\mathrm{U}$ & u & Y & Y & N \\
\hline & 6-Shogaol-S & & Y & Y & Y & $\mathrm{N}$ & $u$ & $u$ & Y & Y & N \\
\hline \multirow[t]{6}{*}{ Marjoram (Origanum onites) } & p-hydroxybenzoic acid & Urine & N & Y & Y & $U$ & U & $u$ & Y & Y & $\mathrm{N}$ \\
\hline & Vanillic acid & & N & Y & Y & $U$ & U & $u$ & Y & Y & N \\
\hline & $m$-hydroxyphenylacetic acid & & $\mathrm{N}$ & Y & Y & $U$ & $U$ & $u$ & Y & Y & N \\
\hline & Ferulic acid & & $\mathrm{N}$ & Y & Y & $U$ & $\mathrm{U}$ & $u$ & Y & Y & N \\
\hline & 3,4-dihydroxyphenylacetic acid & & $\mathrm{N}$ & Y & Y & $U$ & $\mathrm{U}$ & $u$ & Y & Y & N \\
\hline & Protocatechuic acid & & $\mathrm{N}$ & Y & Y & $U$ & $\mathrm{U}$ & $u$ & Y & Y & N \\
\hline
\end{tabular}


Table 3 Summary of the selected candidate BFls of herbs and spices and their assessment relative to the nine validation criteria/ questions described in [33] (Continued)

\begin{tabular}{|c|c|c|c|c|c|c|c|c|c|c|c|}
\hline \multirow[t]{2}{*}{ Food item } & \multirow{2}{*}{$\begin{array}{l}\text { Metabolites } \\
\text { Syringic acid }\end{array}$} & \multirow[t]{2}{*}{ Biofluid locations } & \multicolumn{9}{|c|}{$\begin{array}{l}\text { Questions }^{*} \\
12 \text { 3a 3b } 45678\end{array}$} \\
\hline & & & $\mathrm{N}$ & Y & Y & $\mathrm{U}$ & U & $\mathrm{U}$ & Y & Y & $\mathrm{N}$ \\
\hline & Caffeic acid & & $\mathrm{N}$ & Y & Y & $U$ & $U$ & U & Y & Y & N \\
\hline & p-coumaric acid & & $\mathrm{N}$ & Y & Y & $U$ & $U$ & U & Y & Y & $\mathrm{N}$ \\
\hline \multirow[t]{5}{*}{ Nutmeg } & O-demethyl elemicin & Urine & Y & Y & $\mathrm{N}$ & $U$ & U & Y & U & N & N \\
\hline & O-demethyl dihydroxy elemicin & & Y & Y & $N$ & $U$ & $U$ & Y & $U$ & N & N \\
\hline & Demethylenyl myristicin & & Y & Y & $\mathrm{N}$ & $U$ & U & Y & U & N & N \\
\hline & Dihydroxy myristicin & & Y & Y & $\mathrm{N}$ & $U$ & $U$ & Y & U & N & N \\
\hline & Demethylenyl safrole & & Y & Y & $\mathrm{N}$ & $U$ & $U$ & Y & U & $\mathrm{N}$ & N \\
\hline \multirow[t]{3}{*}{ Parsley } & Apigenin & Plasma & $\mathrm{N}$ & Y & Y & $U$ & $U$ & U & Y & U & N \\
\hline & Apigenin & Red cells & $\mathrm{N}$ & $\mathrm{N}$ & Y & $U$ & U & u & Y & U & N \\
\hline & Apigenin & Urine & $\mathrm{N}$ & Y & Y & $U$ & U & $u$ & Y & Y & N \\
\hline Peppermint (capsule, oral admin.) & Menthol glucuronide & Urine & Y & Y & Y & $U$ & $U$ & U & Y & Y & N \\
\hline \multirow[t]{7}{*}{ Peppermint (intragastric spray) } & Menthol & Plasma & Y & Y & Y & $U$ & $U$ & U & U & Y & $\mathrm{N}$ \\
\hline & Menthol glucuronide & Plasma & Y & Y & Y & $U$ & U & U & Y & Y & N \\
\hline & M7 & Plasma & $U$ & Y & Y & $U$ & $U$ & U & Y & Y & N \\
\hline & M9 & Plasma & $\mathrm{U}$ & Y & Y & $U$ & $U$ & U & Y & Y & N \\
\hline & M11 & Plasma & $\mathrm{U}$ & Y & Y & $U$ & U & $u$ & Y & Y & N \\
\hline & M29 & Plasma & 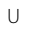 & Y & Y & $U$ & U & U & Y & Y & N \\
\hline & M2-16, M18-21, M24, M29-31 & Urine & $\mathrm{U}$ & $U$ & Y & $U$ & $\mathrm{U}$ & U & Y & Y & $\mathrm{N}$ \\
\hline Saffron & Crocetin & Plasma & Y & Y & Y & $U$ & U & U & Y & Y & N \\
\hline \multirow[t]{10}{*}{ Sage } & 1,8 cineole & Plasma & $N$ & Y & U & $U$ & U & U & $\mathrm{N}$ & Y & N \\
\hline & 1,8 cineole & Urine & $\mathrm{N}$ & Y & $N$ & $U$ & $U$ & U & $\mathrm{N}$ & Y & N \\
\hline & 2-OH-1,8-cineole & Plasma & N & Y & $\mathrm{N}$ & $U$ & U & U & $\mathrm{N}$ & Y & N \\
\hline & 2-OH-1,8-cineole & Urine & $\mathrm{N}$ & Y & $\mathrm{N}$ & $U$ & U & U & $\mathrm{N}$ & Y & N \\
\hline & 3-OH-1,8-cineole & Plasma & $\mathrm{N}$ & $N$ & $N$ & $U$ & U & U & $\mathrm{N}$ & Y & N \\
\hline & 3-OH-1,8-cineole & Urine & $\mathrm{N}$ & Y & $\mathrm{N}$ & $U$ & U & U & $\mathrm{N}$ & Y & N \\
\hline & 7-OH-1,8-cineole & Plasma & $N$ & $\mathrm{~N}$ & $N$ & $U$ & U & U & $\mathrm{N}$ & Y & N \\
\hline & 7-OH-1,8-cineole & Urine & $\mathrm{N}$ & Y & $N$ & $U$ & $U$ & U & $\mathrm{N}$ & Y & N \\
\hline & 9-OH-1,8-cineole & Plasma & $\mathrm{N}$ & Y & $\mathrm{N}$ & $U$ & U & U & $\mathrm{N}$ & Y & $\mathrm{N}$ \\
\hline & 9-OH-1,8-cineole & Urine & $N$ & Y & $N$ & U & U & U & $\mathrm{N}$ & Y & N \\
\hline \multirow[t]{6}{*}{ Tarragon } & Estragole & Plasma & $N$ & Y & Y & $U$ & $U$ & N & U & Y & N \\
\hline & Estragole & Urine & $\mathrm{N}$ & Y & Y & $U$ & U & N & U & Y & N \\
\hline & trans-anethole & Plasma & $N$ & Y & Y & $U$ & U & Y & U & Y & $\mathrm{N}$ \\
\hline & trans-anethole & Urine & $N$ & Y & Y & $U$ & U & Y & U & Y & N \\
\hline & para-Anisaldehyde & Plasma & $\mathrm{N}$ & Y & Y & $U$ & U & Y & U & Y & N \\
\hline & para-Anisaldehyde & Urine & $N$ & Y & Y & $U$ & U & Y & U & Y & N \\
\hline \multirow[t]{13}{*}{ Thyme } & Thymol-S & Plasma & $N$ & Y & Y & $U$ & U & Y & Y & Y & N \\
\hline & Thymol-S & Urine & $\mathrm{N}$ & Y & Y & $U$ & $U$ & Y & Y & Y & N \\
\hline & Thymol-G & Urine & $N$ & Y & Y & $U$ & U & Y & Y & Y & N \\
\hline & Caffeic acid-S & Urine & $\mathrm{N}$ & Y & $\mathrm{N}$ & $U$ & U & Y & Y & Y & N \\
\hline & OH-phenylpropionic acid-S & Plasma & $\mathrm{N}$ & Y & $N$ & $U$ & U & Y & Y & Y & N \\
\hline & $\mathrm{OH}$-phenylpropionic acid-S & Urine & $\mathrm{N}$ & Y & $\mathrm{N}$ & $U$ & $U$ & Y & Y & Y & N \\
\hline & p-cymene-diol-G & Plasma & Y & Y & N & $U$ & U & Y & Y & Y & N \\
\hline & p-cymene-diol-G & Urine & Y & Y & $\mathrm{N}$ & $U$ & U & Y & Y & Y & N \\
\hline & Carvacrol & Feces & $N$ & $\mathrm{~N}$ & $\mathrm{~N}$ & $U$ & $U$ & U & Y & U & N \\
\hline & 2-(4-hydroxyphenyl)acetic acid & Feces & $u$ & N & N & $U$ & $U$ & u & Y & u & N \\
\hline & 3-(3'-4'-dihydroxyphenyl)propionic acid & Feces & $\mathrm{U}$ & N & $\mathrm{N}$ & $U$ & U & U & Y & u & N \\
\hline & OH-phenylpropionic acid & Feces & Y & N & $\mathrm{N}$ & $U$ & $U$ & U & Y & $\mathrm{U}$ & N \\
\hline & 3-phenylpropionic acid & Feces & $U$ & N & $\mathrm{N}$ & $U$ & $\mathrm{U}$ & U & Y & $\mathrm{U}$ & $\mathrm{N}$ \\
\hline Turmeric (curcuminoids in capsule, or nanoemulsion) & Curcumin & Plasma & Y & Y & Y & $U$ & Y & $u$ & Y & Y & $Y$ \\
\hline
\end{tabular}


Table 3 Summary of the selected candidate BFls of herbs and spices and their assessment relative to the nine validation criteria/ questions described in [33] (Continued)

\begin{tabular}{|c|c|c|c|c|c|c|c|c|c|c|c|}
\hline \multirow[t]{2}{*}{ Food item } & \multirow{2}{*}{$\begin{array}{l}\text { Metabolites } \\
\text { Curcumin }\end{array}$} & \multirow{2}{*}{$\begin{array}{l}\text { Biofluid locations } \\
\text { Serum }\end{array}$} & \multicolumn{9}{|c|}{$\begin{array}{l}\text { Questions* } \\
123 \text { a 3b } 45678\end{array}$} \\
\hline & & & Y & Y & Y & U & U & U & Y & $N$ & $\mathrm{~N}$ \\
\hline & Curcumin & Urine & Y & Y & U & U & U & U & Y & U & $\mathrm{N}$ \\
\hline & Curcumin & Feces & Y & Y & U & U & U & U & Y & U & $\mathrm{N}$ \\
\hline & Curcumin & Colon & Y & Y & Y & Y & Y & Y & Y & U & $\mathrm{N}$ \\
\hline & DMC & Plasma & Y & Y & Y & U & Y & U & Y & Y & Y \\
\hline & DMC & Urine & Y & Y & U & U & U & U & Y & U & $\mathrm{N}$ \\
\hline & DMC & Colon & Y & Y & U & Y & U & U & U & U & $\mathrm{N}$ \\
\hline & DMC-G & Plasma & Y & Y & U & Y & U & U & U & U & $\mathrm{N}$ \\
\hline & DMC-G & Urine & Y & Y & U & Y & U & U & U & U & N \\
\hline & DMC-S & Plasma & Y & Y & U & Y & U & U & U & U & N \\
\hline & $\mathrm{BDMC}$ & Plasma & Y & Y & Y & U & Y & U & Y & Y & Y \\
\hline & $\mathrm{BDMC}$ & Urine & Y & Y & U & U & U & U & Y & U & $\mathrm{N}$ \\
\hline & $\mathrm{BDMC}$ & Colon & Y & Y & U & Y & U & U & U & U & N \\
\hline & $\mathrm{THC}$ & Plasma & Y & Y & Y & U & Y & U & Y & Y & Y \\
\hline & COG & Plasma & Y & Y & Y & U & U & U & Y & Y & Y \\
\hline & COG & Urine & Y & Y & U & U & U & U & Y & U & N \\
\hline & COG & Colon & Y & Y & U & Y & U & U & U & U & $\mathrm{N}$ \\
\hline & $\cos$ & Plasma & Y & Y & Y & U & Y & $\mathrm{U}$ & $\mathrm{Y}$ & Y & Y \\
\hline & $\cos$ & Urine & Y & Y & U & U & U & U & Y & U & $\mathrm{N}$ \\
\hline & $\cos$ & Colon & Y & Y & $\mathrm{U}$ & Y & U & U & U & U & N \\
\hline & $\cos$ & Feces & Y & Y & U & U & U & U & $\mathrm{Y}$ & U & $\mathrm{N}$ \\
\hline & Hexahydrocurcumin & Liver & Y & Y & $\mathrm{N}$ & U & U & $\mathrm{U}$ & Y & U & $\mathrm{N}$ \\
\hline & Hexahydrocurcuminol & Liver & Y & Y & $\mathrm{N}$ & U & U & $\mathrm{U}$ & Y & U & $\mathrm{N}$ \\
\hline & Ferulic acid & Plasma & $\mathrm{N}$ & $\mathrm{N}$ & $\mathrm{N}$ & U & Y & U & $\mathrm{N}$ & $\mathrm{N}$ & $\mathrm{N}$ \\
\hline & Vanillic acid & Plasma & N & $N$ & $\mathrm{~N}$ & U & Y & $\mathrm{U}$ & $\mathrm{N}$ & $N$ & N \\
\hline Turmeric (Theracurmin ${ }^{1}$ ) & Curcumin & Plasma & Y & Y & Y & $U$ & U & $\mathrm{U}$ & U & U & N \\
\hline \multirow[t]{3}{*}{ Turmeric-fresh curcuminoids (capsule) } & Curcumin & Plasma & Y & Y & Y & U & U & U & Y & $\mathrm{U}$ & $\mathrm{N}$ \\
\hline & DMC & Plasma & Y & Y & $\mathrm{N}$ & U & U & $\mathrm{U}$ & U & U & N \\
\hline & $\mathrm{BDMC}$ & Plasma & Y & Y & $\mathrm{N}$ & U & $\mathrm{U}$ & $\mathrm{U}$ & U & U & $\mathrm{N}$ \\
\hline \multicolumn{12}{|c|}{$\begin{array}{l}G \text {, glucuronide; }-S \text {, sulfate; } B M D C \text {, bisdemethoxycurcumin; } D M C \text {, demexycurcumin; } D \\
\text { curcumin-O-sulfate. } M 29, \text { menthol sulfoconjugate; hydroxyl menthol; } M 7,9,11 \mathrm{glucuronid} \\
\text { menthol glucuronide; } M 13-18 \text { carboxylate-menthol, or aldehyde-hydroxyl menthol glucur } \\
\text { 'Theracurmin, commercialized nanoparticle curcumin to increase its absorption } \\
\text { "Possible answers are } Y \text { (yes), } N \text { (no), or } U \text { (unknown or uncertain, or not specified in the } \\
\text { Biological/nutritional validation and applicability: } \\
\text { 1. Is the marker compound known as unique for the food or food group (chemical/bioloc } \\
\text { 2. Is there a dose-response relationship at relevant intake levels of the targeted food (qua } \\
\text { 3a. Is the single-meal time-response relationship described adequately (single-dose kineti } \\
\text { 3b. Is the biomarker kinetics for repeated intakes of the food/food group adequate (e.g., } \\
\text { 4. Has the marker been shown to be robust after intake of complex meals (robustness)? } \\
\text { 5. Has the marker been shown to compare well with other markers for the same food/foc } \\
\text { Analytical validation: } \\
\text { 6. Is the marker chemically and biologically stable, making measurement reliable (feasibil } \\
\text { 7. Are analytical variability (CV\%), accuracy, sensitivity, and specificity known as adequate } \\
\text { 8. Has the analysis been successfully reproduced in another laboratory (reproducibility)? }\end{array}$} \\
\hline
\end{tabular}

consumption. The authors concluded that anethole can be reliably detected in blood/serum samples after consumption of spirits containing anethole. In no case was a positive result for anethole found where $40 \mathrm{ml}$ or less of spirits containing anethole had been consumed or where the time difference between the cessation of drinking and the taking of the blood sample was greater than $4 \mathrm{~h}$.

Based on these two studies, we can conclude that anethole seems to be a robust and reliable blood BFI for anise consumption as assessed by observational studies involving the consumption of anise-based drinks. While a single, high-quality marker for specific food intake is ideal, the addition of other (unrelated) biomarkers to create a multi-component biomarker panel can substantially improve a biomarker's sensitivity and specificity [33]. In this regard, two compounds, 4-methoxyhippuric acid and 4-methoxybenzoic acid have been specifically detected in urine after direct anethole intake. However, these two compounds are actually metabolites of anethole and so are unlikely to add to anethole's sensitivity/ 


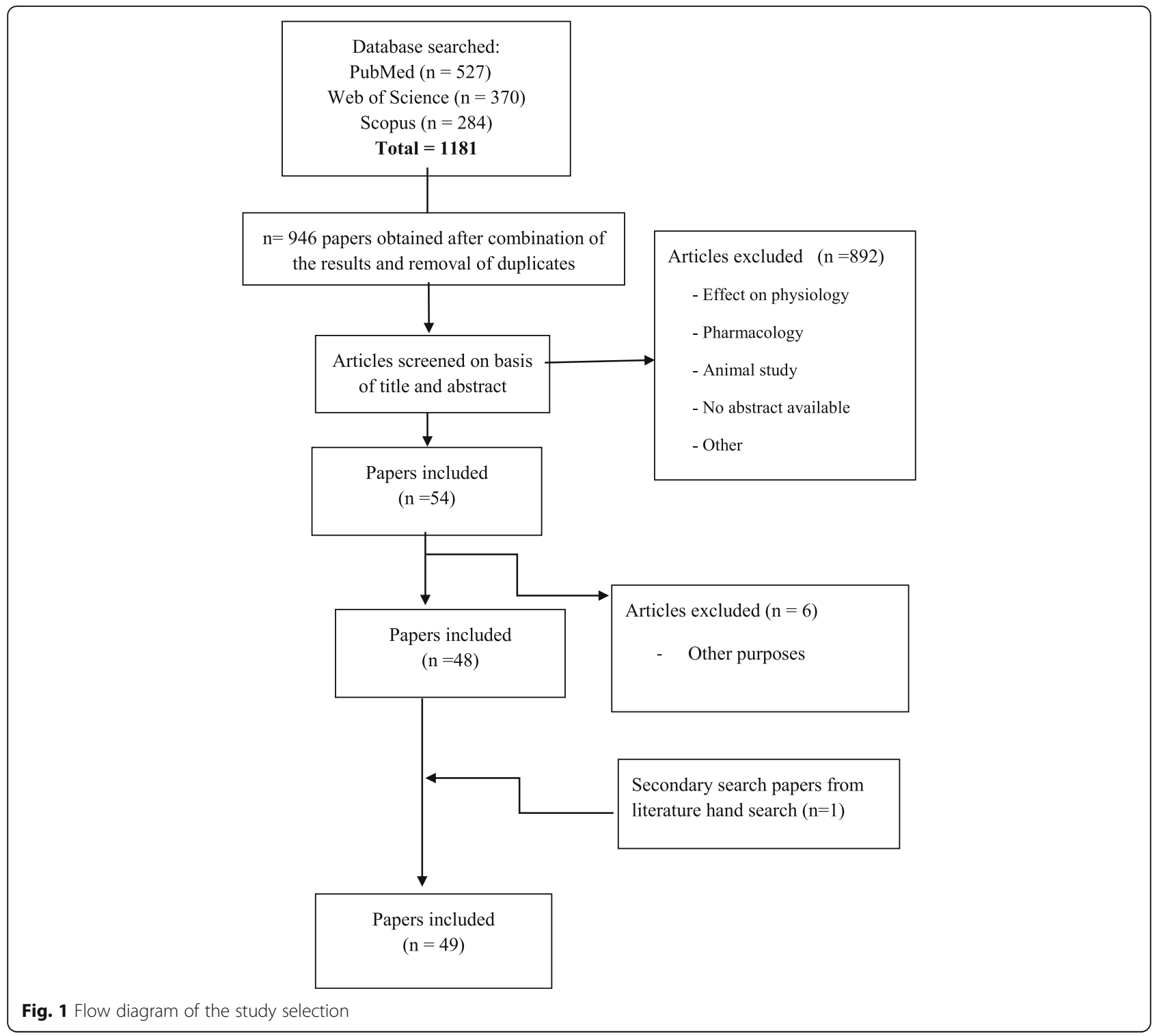

specificity. We also believe that further studies are needed to confirm that these two compounds are seen with actual anise-based food intake. It is also worth noting that anethole is found in fennel, basil, and tarragon [38], and it is widely present in pharmaceutical products and as a flavoring additive. Therefore, anethole and its metabolites may not be sufficiently specific BFIs for anise intake.

\section{Capsicum sp.}

\section{Chili pepper}

The chili pepper is a fruit spice derived from plants from the genus Capsicum, originated in Mexico and brought to Asia by Portuguese navigators during the sixteenth century. The five domesticated species of pepper are Capsicum annuum, C. frutescens, C. chinense, C. pubescens, and C. baccatum. Chili peppers have a taste that is pungent, hot, and somewhat sweet (depending on the variety and type). Mild or sweet peppers contain similar constituents as Capsicum but with little or no pungent components. Chili peppers are used as food colorants, flavoring agents, as predator repellants, and a source of pain relief. The compounds responsible for the "hot" flavor of chili peppers are called capsaicinoids, with capsaicin being the best known. Capsaicin occurs naturally in plants of the Solanaceae family. It is commonly used in both food and medicine, but its strong pungency limits the quantity that can be employed. Capsicum contains up to $1.5 \%$ (by weight) of pungent compounds, commonly composed of capsaicin, dihydrocapsaicin, and others. Other constituents present in chili peppers are carotenoids, vitamins A, C, and small amounts of volatile oils with more than 125 known 


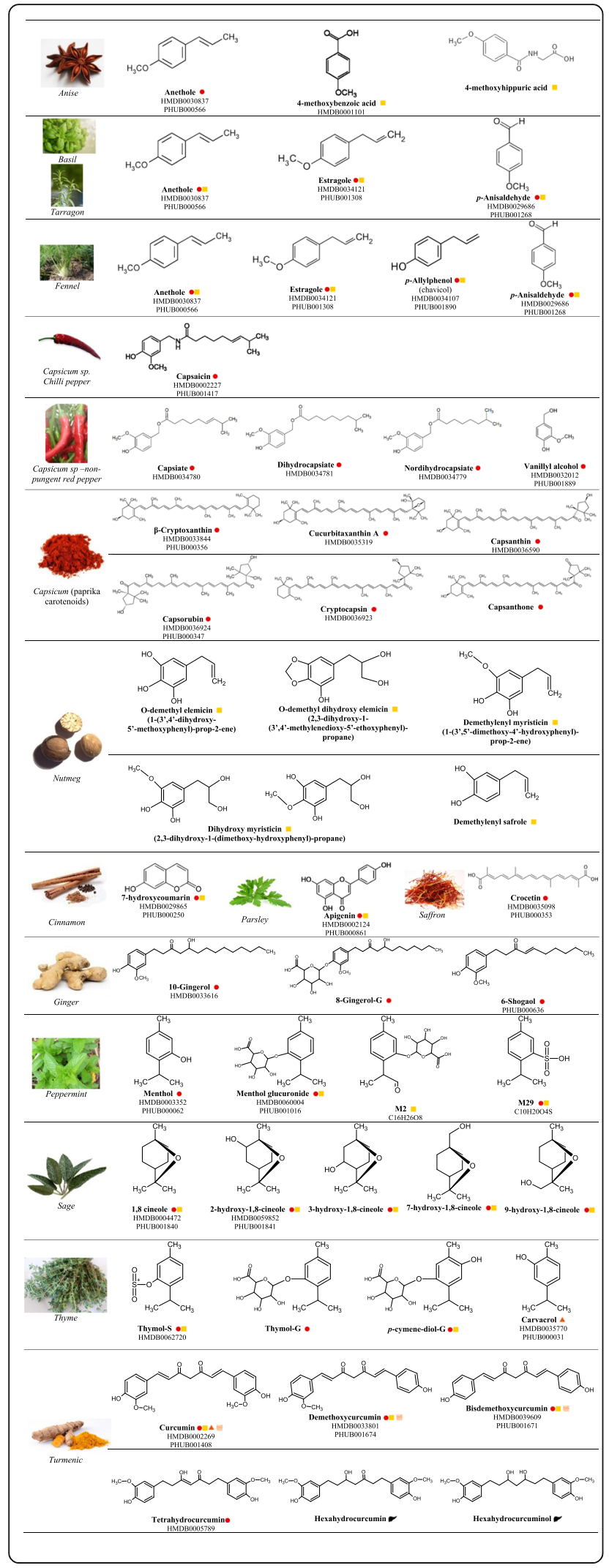

Fig. 2 Examples of the most representative metabolites for each herb/spice identified in the reviewed studies. HMDB ID; phytohub code (PHUB); -G, glucuronide; $-S$, sulfate; aldehyde-menthol glucuronide (M2); menthol sulfate conjugates (M29); in the blood; in urine; $\boldsymbol{\Delta}$ in feces; in the intestine;

components. Another class of capsaicin-like compounds found in chili peppers and non-pungent chili peppers are the capsinoids. Capsinoids have an estimated "hot taste threshold" that is about $1 / 1000$ that of capsaicin making it possible to use capsinoids in food applications without the intense heat effect found in capsaicins [39]. Many positive health benefits have been ascribed to both capsaicin and capsinoids, including anticancer, anti-inflammatory, and analgesic effects [40].

We found two published studies that explored possible BFIs associated with chili pepper consumption. In a cross-over study with 12 volunteers [41], capsaicin was detected in plasma by HPLC analysis after the administration of $5 \mathrm{~g}$ of capsaicin derived from chili pepper. This pharmacokinetic study showed that capsaicin was rapidly absorbed (being detected at $10 \mathrm{~min}$ ) after ingestion and also rapidly metabolized (not detected in blood after $90 \mathrm{~min}$ ). Another study, conducted by Bernard et al. [42], analyzed the metabolites present in plasma by LC-MS/MS after the administration of a capsule of a variety of non-pungent (sweet) pepper (CH-19) extract. The compounds identified were the capsinoids capsiate, dihydrocapsiate and nordihydrocapsiate, and a capsinoid metabolite, vanillyl alcohol. However, these compounds were below the limit of quantification, so the authors could not perform proper kinetic studies.

Based on these data, capsaicin, the main compound responsible for the hot taste of chili peppers, can be considered a specific BFI for chili peppers. However, we believe more data are required and further studies should be performed to confirm the utility of capsaicin as a BFI. With regard to the non-pungent compounds of the "sweet" varieties of Capsicum, (i.e., capsinoids) additional dose-response studies are needed to consider them as plausible BFIs since the concentrations measured in the single reported study were too low to perform kinetic analyses.

\section{Paprika}

Paprika is a ground spice made from the red, air-dried fruits of the larger and sweeter varieties of the plant Capsicum annuum, which is also called bell pepper or sweet pepper. Paprika can also be modified with the addition of more pungent chili peppers and cayenne pepper. Originating in central Mexico, paprika was brought to Spain in the sixteenth century. Paprika spices can range from mild to hot, depending on the variety of the source plant. The flavor also varies from country to country-but almost all plants grown produce the sweet variety. The red, orange, 
or yellow color of paprika is due to its content of carotenoids. The intense color of paprika makes it an ideal and natural food colorant for many dishes.

Only one study was found that looked into the identification of biomarkers of food intake for paprika. Nishino and co-workers [42] measured paprika carotenoids in the plasma and erythrocytes of five volunteers who were supplemented with paprika for 4 weeks. The results showed that several, non-unique carotenoids such as lutein, zeaxanthin, $\beta$-cryptoxanthin (also found in vegetables such as carrots and tomatoes) could be detected in the paprika-supplemented volunteers. However, the authors also detected other carotenoids specific for paprika, such as cryptocapsin, capsanthin, capsorubin (just found in paprika and lily pollen), cucurbitaxanthin A (found just in paprika and pumpkins), and finally capsanthone (a possible oxidative product of capsanthin). Paprika carotenoids, particularly capsanthin and capsorubin, have been reported to have a strong antioxidant activity [43, 44]. Based on these results, cucurbitaxanthin A, capsanthin, capsanthone, and cryptocapsin could be potential paprika-specific carotenoid biomarkers. However, further analyses using untargeted MS-based approaches should be conducted to evaluate other possible biomarkers of paprika intake.

\section{Cinnamon}

Cinnamon is a bark spice obtained from the inner bark of several tree species from the genus Cinnamomum. Only a few Cinnamomum species are grown commercially (largely from Asia) for spice. Cinnamon is native to India, Sri Lanka, Bangladesh, and Myanmar, and it was imported to Egypt as early as 4000 years ago [45]. In addition to its common culinary use as a condiment and flavoring material, cinnamon is widely known for its anti-diabetic and glucose lowering effects [46]. The flavor of cinnamon is due to an aromatic essential oil that is largely composed of cinnamaldehyde (up to 90\%); however, there are at least 80 other compounds known to be in cinnamon oil, including cinnamyl alcohol, cinnamyl acetate, eugenol, and various coumarins that contribute to its overall flavor and aroma [47].

Only one study has been performed in humans to identify BFIs of cinnamon intake [48]. While cinnamaldehyde might have been expected to be a useful BFI, it is quickly metabolized to cinnamic acid [49], making it unusable as a cinnamon biomarker. On the other hand, coumarin was deemed to be a potentially useful BFI for cinnamon. Because coumarin has a very strong first-pass effect in the liver, with only a small percentage reaching systemic circulation, the authors chose its main metabolite, 7- hydroxycoumarin as a measure of relative bioavailability. In the study by Abraham et al. [40], 7-hydroxycoumarin was assessed as a biomarker of cinnamon consumption in both urine and plasma via HPLC-MS/MS analysis. The conversion of coumarin to 7-hydroxycoumarin is catalyzed by cytochrome P450 2A6 (CYP2A6) [50]. 7-hydroxycoumarin and its phase II metabolite, 7-hydroxycoumarin glucuronide, are rapidly excreted via the kidneys [51]. Therefore, the total amount of 7-hydroxycoumarin (free and bound as a glucuronide) in urine could serve as an indirect measure of the extent of cinnamon consumption.

Coumarin possesses a pleasant spicy odor of fresh hay or vanilla [48]. The occurrence of coumarin has been reported in a number of bedding plants such as Anthoxanthum odoratum (sweet vernal grass), Asperula odorata (sweet woodruff), Dipterix odorata (tonka bean), Eupatorium triplinerve (white snakeroot), Hierochloe odorata (holy grass), Melilotus coerulea (sweet trefoil), M. officinalis (common melilot), Melittis melissophyllum (bastard balm), Primula elatior (oxlip), and Trilisa odoratissima (deer tongue). However, none of these plants are usually used as edible foods; thus, the main source of coumarin in the diet is cinnamon [52]. Coumarin, which is frequently used in perfumes, is also a well-known hepatotoxin (based on animal studies). Interestingly, different species of cinnamon have different levels of coumarin. For example, $C$. cassia cinnamon contains up to $1 \%$ coumarin, whereas the more expensive and less frequently used true cinnamon (Cinnamomum verum) contains only trace levels $(0.004 \%)[53,54]$. Today, many commercially available food products are spiced with the cheaper $C$. cassia cinnamon and consequently contain high levels of coumarin. It is notable that German Christmas cookies (which contain considerable amounts of $C$. cassia cinnamon) have a coumarin content that often exceeds the maximum tolerable dose intake (TDI $0.1 \mathrm{mg} / \mathrm{kg}$ body weight).

Based on the available data and based on the fact that the food matrix effect has been well tested, 7-hydroxycoumarin appears to be a plausible specific and robust biomarker of cinnamon intake. Additional cumulative/kinetic aspects of this biomarker need to be performed and most likely an inter-laboratory validation needs to be completed to fully validate this compound as a cinnamon BFI. However, the use of this metabolite as a BFI for cinnamon is confounded by the fact that it depends on the species of cinnamon being used. We believe that other cinnamon compounds (such as cinnamaldehyde and cinnamic acid or the essential oils as cinnamyl alcohol, cinnamyl acetate and eugenol)) should also be explored as potential biomarkers as, so far, the only reported cinnamon BFI study was limited to measuring coumarin and its derivatives.

\section{Fennel, basil, and tarragon}

While fennel, basil, and tarragon are very distinct herbs, coming from very different plant species, they share a 
number of common chemicals and consequently they tend to be grouped together in food intake studies. This is why we have chosen to group these three herbs under a single topic heading.

Fennel is a seed (and bulb) spice, as well as a leaf herb, that is derived from Foeniculum vulgare. This is a small flowering plant that was originally indigenous to the shores of the Mediterranean, but which has since become widely naturalized in many parts of the world. Fennel is a highly aromatic and flavorful herb/spice and is one of the primary ingredients of absinthe. The distinctive licorice flavor and aroma from fennel comes from anethole. Other compounds known to be in fennel include estragole, fenchone, 1,8-cineole (eucalyptol), and $p$-allylphenol. In addition to its use in culinary applications, fennel has long been used as a medicinal herb to treat gastrointestinal illnesses and upper respiratory tract infections as well as to increase milk production in breastfeeding mothers through the consumption of fennel tea.

Basil (Ocimum basilicum) is a culinary herb belonging to the botanical family Lamiaceae. It is a culinary herb that is prominently featured in Italian cuisine as well as many Southeast Asian cuisines. Depending on the species and cultivar, the leaves may taste somewhat like anise, with a strong, pungent, often sweet smell. Thai basil is also a condiment in the Vietnamese noodle soup. Basil has been used traditionally as a medicinal herb in the treatment of headaches, coughs, diarrhea, constipation, warts, worms, and kidney disorders [55]. It is also a source of aroma compounds and essential oils containing biologically active constituents that possess antimicrobial and antifungal properties [56, 57]. Linalool is the main constituent of the essential oil of $O$. basilicum (28.6-60.6\%), followed by estragole, methyl cinnamate, epi- $\alpha$-cadinol, $\alpha$-bergamotene, $\gamma$-cadinene (3.3-5.4\%), germacrene $\mathrm{D}$ (1.1-3.3\%), and camphor (1.1-3.1\%). Other compounds such as myrcene, pinene, terpineol, 1,8-cineole, eugenol, and methyleugenol have been identified in basil leaves [56, 58,59].

Tarragon (Artemisia dracunculus), also known as estragon, is a perennial herb belonging to the Asteraceae (daisy) family. It is widespread across much of Eurasia and North America, and is cultivated for culinary and medicinal purposes. Two well-described "cultivars" (Russian and French) are widely used. "Dracunculus" which in Latin meaning "little dragon" is believed to describe its coiled, serpentine root, and/or the shape of the leaves, which is reminiscent of a dragon's tongue [60]. In vitro pharmacological studies indicate that tarragon has antibacterial, antifungal, and antiplatelet activity [61]. In vivo pharmacological studies have shown that tarragon has anti-inflammatory, hepatoprotective, antihyperglycemic, and antioxidant activity [61]. The major components of Russian tarragon are reported to be terpinen-4-ol, sabinene, and elemicin. Methyleugenol and estragole are usually present in tarragon oils at about 10 and $3 \%$, respectively. However, estragole is one of the predominant compounds in the essential oil of French tarragon, constituting up to $82 \%$ [61]. Trans-anethole (21.1\%), $\alpha$-trans-ocimene (20.6\%), limonene (12.4\%), $\alpha$-pinene (5.1\%), and allo-ocimene (4.8\%) are the other main components of tarragon [62].

Two studies have explored or assessed potential biomarkers of fennel (alone) or fennel, tarragon, and basil intake in humans. The first one, by Zeller et al. [63], studied the metabolism of estragole in humans consuming fennel tea. The metabolites identified in the urine of subjects were estragole, 1'-hydroxyestrogle, trans-anethole (also reported in anise $[36,37]$ ), and $p$-allylphenol (also found in betel leaf oils and in oil of bay). However, the authors were unable to report concentrations for these compounds or to correlate them with fennel dosage. In terms of the specificity of these compounds, estragole, in addition to being found in fennel, is a known component of several herbs such as tarragon, basil, and anise. Estragole, which is structurally similar to safrole, is rapidly metabolized to 1 '-hydroxyestragole and is quickly excreted as its glucuronic acid conjugate.

In the second study by Barfi et al. [38], the authors developed and validated a multi-step method to extract trans-anethole, estragole, and para-anisaldehyde (three major components of fennel, basil and tarragon) from biofluids and then applied this extraction technique to real human plasma and urine samples. All three compounds were found in plasma and urine after the consumption of either $15 \mathrm{ml}$ of fennel extract, $15 \mathrm{ml}$ of tarragon extract or $15 \mathrm{ml}$ of brewed basil.

While studies of fennel, basil, and tarragon phytochemicals and essential oils have identified several potentially unique compounds for each of these herbs, the same cannot be said of the BIFs that have been, so far, identified. To date, all of the food intake compounds identified for fennel, basil, and tarragon consumption $[38,63]$ are not sufficiently specific to identify one from the other or any of them from other widely consumed herbs. This is because all of the reported compounds found in human biofluids, so far, are also found in other herbs and spices (such as anise). Therefore, we conclude that no specific BFI for fennel, basil, or tarragon intake has, to date, been discovered or described in the literature.

\section{Ginger}

Ginger (Zingiber officinale) is a root or rhizome-based spice derived from the ginger plant, a member of the turmeric family (both are from Zingiberaceae). Ginger is believed to have originated in India and is widely used as 
a culinary additive as a hot, fragrant spice as well as a popular medicine. In addition to ginger's well-known use as a treatment for nausea, many components in ginger have been found to have anti-inflammatory, antibacterial, antipyretic, antilipidemic, antitumorigenic, and antiangiogenic effects [64-66]. Ginger's flavor and aroma come from its volatile oils ( $\sim 1$ to $3 \%$ of the weight of fresh ginger) and non-volatile pungent oleoresins. A variety of active components have been identified in the oleoresins of ginger including zingerone, gingerols (6-, 8-, and 10-gingerols), and shogaols (6-, 8-, and 10-shogaols) [67]. Gingerols (especially 6-gingerol) are the major pungent components in the fresh ginger rhizome. In dried ginger, the quantity of shogaols are significantly increased as evidenced by the reduction of the ratio of 6-gingerol to 6-shogaol from 10:1 in fresh ginger to $1: 1$ in dried ginger [68]. In particular, zingerone is produced from gingerols during drying, having lower pungency and a spicy-sweet aroma.

A total of three studies have been reported on potential BFIs for ginger or ginger extracts. The earliest study by Zick et al. [69] found that 6, 8-, and 10-gingerols and 6-shogaol are absorbed after oral ginger extract dosing and can be detected as glucuronide and sulfate conjugates [69]. $\mathrm{Yu}$ and co-workers [55] detected free 10-gingerol and 6-shogaol in the human plasma, whereas the majority of the 6-, 8-, and 10-gingerols and 6-shogaol existed as glucuronide and sulfate metabolites after oral dosing of $2 \mathrm{~g}$ ginger extracts [70]. No free 6-gingerol was detected in plasma despite it being the most abundant component of ginger extracts (2.64\%). In comparison, although 6-shogaol makes up $2.25 \%$ and 10-gingerol only accounts for $1.22 \%$ of most ginger extracts, 6-shogaol and 10-gingerol were readily detected in human plasma. Pharmacokinetic studies showed very short half-lives for these four analytes and their metabolites $(1-3 \mathrm{~h}$ in human plasma). Due to their short half-lives, no accumulation was observed for 6-, 8-, and 10-gingerols and 6-shogaol (and their metabolites) in either plasma or colon tissues even after multiple daily dosing. A third biomarker intake study was focused on the metabolism of shogaol [60]. The results of this study (Table 2) show that it was possible to detect all the major thiol-conjugated metabolites of shogaol in human urine using LC-MS/MS [71]. The authors suggested the mercapturic acid pathway as a major metabolic route for shogaols in humans.

Based on the available data, 6-, 8-, and 10-gingerol glucuronides and sulfates along with 6-shogaols appear to be plausible, specific, and robust biomarkers of ginger intake. More studies are needed to confirm that they are also seen with actual ginger-based food intake. Additional cumulative/kinetic aspects of these biomarkers need further evaluation and most likely an inter-laboratory validation is required to make these compounds fully validated BFIs for ginger consumption.

\section{Nutmeg}

Nutmeg is a fragrant flavoring spice coming from the seed of Myristica fragrans (belonging to the Myristicaceae family), an evergreen tree indigenous to the Banda Islands in the Moluccas (or Spice Islands) of Indonesia. Until the mid-nineteenth century, the small island group of the Banda Islands, was the only location of the production of nutmeg and mace in the world. This made nutmeg a particularly prized and costly spice in European medieval cuisine. The nutmeg essential oil is obtained by steam distillation of ground nutmeg, and it is used widely in the perfumery and pharmaceutical industries. This volatile fraction typically contains sabinene (21.38\%), 4-terpineol (13.92\%), and myristicin (13.57\%), as well as portions of safrole, elimicin, terpineol, $\alpha$-pinene $\mathrm{d}$-camphene, limonene, linalool, and isoeugeunol [72]. Psychotropic effects have been described after ingestion of large doses of nutmeg, which are attributable to metabolic formation of amphetamine derivatives from the main nutmeg ingredients elemicin, myristicin, and safrole.

Only one study was found for the evaluation of nutmeg ingestion. Beyer and co-workers [73] evaluated nutmeg administration in animals and then performed an observational toxicological study to identify the metabolites in the urine of a human subject after the individual ingested the powder derived from 5 nutmeg seeds. In the human urine sample, the following metabolites were identified by GC-MS: O-demethyl elemicin, O-demethyl dihydroxy elemicin, demethylenyl myristicin, dihydroxy myristicin, and demethylenyl safrole. Neither amphetamine derivatives nor the main nutmeg ingredients could be detected in the rat urine nor in human urine samples [73].

Myristicin is a natural organic compound not only present in nutmeg oil, but also, to a lesser extent, in members of the Umbelliferae family such as carrots, parsley, celery, dill, parsnip, and black pepper [74]. The measured amount of myristicin in nutmeg and mace is very high $-13,000 \mathrm{mg} / \mathrm{kg}$ (nutmeg) and $27,000 \mathrm{mg} / \mathrm{kg}$ (mace). On the other hand, it is much less in dill and parsley $(1200 \mathrm{mg} / \mathrm{kg}$ and $727 \mathrm{mg} / \mathrm{kg}$, respectively), and very low in celery $(0.33 \mathrm{mg} / \mathrm{kg})$, carrots $(0.16 \mathrm{mg} / \mathrm{kg})$, and parsnip $(0.002 \mathrm{mg} / \mathrm{kg})$ [74]. Zheng et al. [75] found that in an in vivo animal study conducted on mice, myristicin had the ability to increase the activity of the detoxifying system (potential cancer chemoprevention). This finding was replicated in another study [76]. Elemicin has been identified as an essential oil composition of carrots [77], parsley, elemi oil, banana, anise, and oregano [78]; however, the major route of elemicin intake 
appears to be nutmeg [79]. Lastly, safrole is a major chemical constituent (85\%) of the aromatic oil of sassafras root bark (Sassaras albidum). It is also a minor component or trace constituent in mace, nutmeg, cinnamon, black pepper, cocoa, anise, and a number of other spices [80].

Because nutmeg is the primary known dietary source of these compounds [81], many of the metabolites of myristicin, elemicin, and safrole either alone or in combination (as a multi-marker panel) could be considered as good candidate BFIs of nutmeg intake. However, since the only study that exists on nutmeg intake is an observational study, additional controlled kinetic studies should be conducted, and further analytical performance validation should be done before these BFIs can be fully validated.

\section{Oregano, marjoram, rosemary, and thyme}

Oregano, marjoram, rosemary, and thyme are culinary herbs derived from members of the Lamiaceae plant family, which also includes basil, mint, sage, lavender, and others. Due to their phylogenetic proximity and the similarity of the compounds identified in studies of these herbs, we decided to present the results and discuss them together.

Oregano (Origanum vulgare) is a native herb to temperate western and southwestern Eurasia and the Mediterranean region. It has an aromatic, warm, and slightly bitter taste. Among the chemical compounds contributing to the flavor of oregano are carvacrol, thymol, limonene, pinene, ocimene, and caryophyllene [82]. Oregano also contains polyphenols, including caffeic, $p$-coumaric, and rosmarinic acid, which confer antioxidant activity and prevents lipid peroxidation [4]. It is widely used in Mediterranean cuisine, the Philippines, and Latin America, especially in Argentina. A related herb from Origanum oonites, which is better known as marjoram, is a plant species found in Sicily, Greece, and Turkey. Marjoram has similar flavors as oregano.

Rosemary (Rosmarinus officinalis) is native to the Mediterranean and Asia. The leaves are used as a flavoring agent in a variety of foods in traditional Mediterranean cuisine. They have a bitter, astringent taste, and a very characteristic aroma. Rosemary contains a number of phytochemicals, including rosmarinic acid, camphor, caffeic acid, ursolic acid, betulinic acid, carnosic acid, and carnosol [83]. Major essential oils present in rosemary oil are borneol (26.5\%), $\alpha$-terpinene $(15.6 \%)$, and $\alpha$-pinene (12.7\%) [84].

Thyme (Thymus vulgaris) is also a member of the Lamiaceae family, and it has been used in foods mainly for flavor, aroma, and food preservation. Thyme has also been used in folk medicine since the times of the ancient Egyptians, Greeks, and Romans. The leafy parts of thyme are often added to meat, fish, and food products and also used as herbal medicinal products. The essential oils of common thyme contain $20-58 \%$ thymol and $p$-cymene $(15-28 \%)$ as the most prevalent compounds, followed by linalool (0.7-6.5\%), $\gamma$-terpinene $(4-10 \%)$, carvacrol (1-4\%), myrcene (1-3\%), 1,8-cineole $(0.8 \%)$, and borneol $(0.7-1.7 \%)$ [85-87]. Thymol is the compound that provides the distinct flavor of thyme. It is also found in oregano and is used as one of many additives in cigarettes.

Oregano, rosemary, thyme, along with sage and mint are known to share several polyphenols and essential oils. Shared polyphenols include caffeic acid, chlorogenic acid, ferulic acid, $p$-coumaric acid, $p$-hydroxybenzoic acid, protocatechuic acid, and rosmarinic acid [83]. Some of the essential oils that are common to many herbs in the Laminaceae family are thymol (thyme, oregano, marjoram), carvacrol (thyme, oregano, marjoram), carnosic acid (rosemary and sage), carnosol (rosemary and sage), and rosmanol (rosemary and sage) [4].

Oregano, thyme, and rosemary are well known for their beneficial health properties. For example, carnosic acid and some of the diterpenes abundant in rosemary and sage appear to exert anti-obesity effects (including body weight and lipid-lowering effects) [88]. Likewise, thymol and carvacrol (oregano, thyme), carnosic acid, carnosol, rosmanol, (rosemary, sage), and epirosmanol (rosemary) have been shown to prevent lipid peroxidation and to have anti-inflammatory activity $[4,89-94]$. Rosmarinic acid (found in oregano, sage, basil, rosemary, thyme and mint) exhibits anti-inflammatory effects while ferulic acid, caffeic acid, and $p$-coumaric acid inhibit LDL peroxidation [4]. Several compounds found in herbs from the Laminaceae family also exhibit antimicrobial activity, such as thymol, carvacrol, carnosol, rosmanol, and caffeic acid [7].

With regard to BFIs for these four herbs (thyme, marjoram, oregano, rosemary), a total of four studies were found that evaluated thyme intake, one study was found for marjoram intake, and one for oregano (evaluated in mice) and one for rosemary (evaluated in rats). In terms of oregano BFI intake, carvacrol, one of the principal components of oregano, was detected by LC-MS/MS in both murine plasma and brain after oregano extract administration [95]. Carvacrol was also found after ingestion of thyme, in a study that involved two different experimental models: (1) in vitro fermentation and (2) human intervention (in feces) [96]. These authors found that the in vitro fermentation showed limited degradation of thymol and carvacrol while the human intervention study, which used thyme phenol-enriched olive oil, increased the levels of phenylpropionic and hydroxyphenylpropionic acids in human feces, confirming in vivo microbial degradation of rosmarinic acid and eriodictyol. 
Based on these data, carvacrol can be considered a specific biomarker of thyme and oregano intake.

A human study looking at BFIs for marjoram measured urinary metabolites of 6 healthy volunteers, each of whom took a single dose of marjoram extract. In this study, the urinary metabolites identified by an HPLC-coulometric electrode array detector (CEAD) [97] were mainly polyphenol compounds such as protocatechuic acid, $p$-hydroxybenzoic, caffeic, ferulic, syringic, vanillic, $p$-coumaric, 3,4-dihydroxyphenylacetic, $m$-hydroxyphenylacetic acids. These polyphenols are also found in many other plant foods and beverages such as tea, wine, coffee, cereals, cocoa, and in general in vegetables and fruits (http://phenol-explorer.eu).

In the BFI evaluation studies of thyme, thymol [96, 98], and its derived metabolites (thymol sulfate, thymol glucuronide) [99], along with a number of polyphenols and polyphenolic metabolites including hydroxyphenyl propionic acid sulfate, coumaric acid sulfate, caffeic acid sulfate, ferulic acid sulfate, hydroxybenzoic acid dihydrophenylpropionic acid sulfate were identified in both rats [98] and humans [99]. Rosmarinic acid was also detected in plasma after thyme administration in a controlled randomized trial performed on Wistar rats [98]. Rosmarinic acid is found in both thyme and rosemary and can be considered as a biomarker of intake for both spices. Romo-Vaquero and collaborators [100] evaluated the gut, liver, plasma, and small intestine of 24 Zucker rats after both acute and subchronic administration of rosemary extract. Although the main bioactive compound of rosemary is carnosic acid [101], other derived metabolites (shown in Table 2) were also detected in this Zucker rat study. Because the two studies we found for rosemary and oregano administration were performed on animals, the compounds identified are simply being reported here for completeness. The data cannot be used to infer human BFIs for these two herbs. More studies in humans for rosemary and oregano are needed to better understand the metabolites and establish a set of specific BFI for humans.

Based on the data described here, we can conclude that several polyphenols and essential oils are shared between oregano, marjoram, rosemary and thyme. Most of these polyphenols are also common to other fruits and vegetables, so they cannot be considered specific biomarkers for these herbs. While rosmarinic acid is a very specific polyphenol for this family (Laminaceae), no rosamarinic acid could be detected in humans after consumption due to its rapid metabolism. With regard to essential oils, thymol and thymol sulfate in plasma and urine and thymol glucuronides in urine seem to be plausible biomarkers of thyme intake. However, these compounds are also present in oregano. Given the rather sparse number of studies done in humans for these four herbs, it is clear that additional human intervention studies are needed to evaluate if several compelling candidate biomarkers (i.e., carvacrol, carnosic acid, carnosol, rosmanol and derivatives) seen in animals can also be confirmed in human studies.

\section{Parsley}

Parsley (Petroselinum crispum) is an herb belonging to the Apiaceae family. It is native to the central Mediterranean region. Fresh parsley has a clean, green aroma with a versatile fresh taste that is slightly peppery with an aftertaste of green apple. Parsley is a source of several flavonoids, especially luteolin and apigenin [102, 103]. Apigenin is associated with anti-inflammatory activities as it appears to downregulate or inhibit cyclooxygenoase-2 (COX-2) [104]. Apigenin has also been identified as a potential cancer chemopreventive agent [105]. The major essential oil found in parsley leaves is 1,3,8-p-menthatriene, but other components are also present in lesser amounts including myristicin and limonene, among others $[106,107]$.

Two research articles were found that focused specifically on measuring BFIs of parsley [108, 109]. The first article focused on the development of a method to analyze apigenin and the apigenin metabolite acacetin (4'-methoxyapigenin) in human samples using an HPLC-UV method [108] while the second article focused on an intervention study [109] that measured these two compounds after the consumption of parsley. In this randomized crossover trial, parsley consumption together with a low flavone diet revealed a strong correlation with urinary apigenin excretion [108]. In addition to these two BFI parsley studies, several other studies have also assessed apigenin intake. One study [102], which looked at the ingestion of apigenin-rich foods like parsley, found that apigenin was elevated in urine, plasma, and red blood cells (RBCs). Although apigenin could be identified in plasma and RBCs, it was barely detectable (i.e., close to the limit of detection). On the other hand, apigenin in urine was easily identified in all individuals [102]. In another study that used a randomized crossover design for the administration of parsley, apigenin could not be detected in plasma because it was below the lower the limit of detection for the type of assay and instrument used [110].

Apigenin is a flavone not only found in parsley, but also in other vegetables from the same family (Apiacea), such as celery, and in lesser amounts in parsnip, carrots, and fennel. So while parsley certainly has a high content of apigenin, due to the poor specificity of this polyphenolic compound, we conclude that apigenin would not be a suitable BFI of parsley intake. Therefore, we conclude that there are no useful BFIs of parsley intake 
that have yet been discovered or reported in the literature.

\section{Peppermint}

Peppermint and spearmint are herbs that belong to the Laminacea family. Spearmint (Mentha spicata) is believed to be the oldest of the mints. It is a species of mint native to much of Europe and Asia (the Middle East, the Himalayas, China) that is now found in many parts of Northern and Western Africa, North America, and South America. On the other hand, peppermint (Mentha $\times$ piperita) is a hybrid mint herb. In particular, it is a cross between watermint and spearmint, and is indigenous to Europe and the Middle East. Both spearmint and peppermint have a fresh, minty, weedy, aroma. The taste is spicy, minty cool, sweet, and slightly pungent [111]. The active constituents of spearmint include spearmint oil, various flavonoids (diosmin, diosmetin), phenolic acids, and lignans. The most abundant compound in spearmint oil is carvone, which gives spearmint its distinctive smell. Spearmint oil also contains significant amounts of limonene, dihydrocarvone, and 1,8-cineol [112]. Unlike peppermint oil, spearmint oil contains minimal amounts of menthol and menthone. Conversely, peppermint has a high menthol content (40.7\%), along with menthone (23.4\%), and other essential oils such as menthyl acetate (4.2\%), 1,8-cineole (5.3\%), limonene (2.6\%), menthofuran (3.7\%), and $\beta$-caryophyllene $(1.7 \%)[113,114]$. Peppermint leaves are often used alone or with other herbs in herbal teas (tisanes, infusions), ice cream, confectionery, chewing gum, toothpastes, and shampoos. Menthol activates cold-sensitive receptors in the skin and mucosal tissues, and is the primary source of the cooling sensation that follows the topical application of peppermint oil [115]. Peppermint also contains terpenoids and flavonoids such as eriocitrin, hesperidin, and kaempferol 7-O-rutinoside.

Interestingly, six research papers were found that studied the administration of peppermint (oil), but no studies were found that assessed spearmint administration. All six peppermint studies evaluated menthol and its glucuronide conjugate after administration of peppermint oil using a targeted approach. The main goal of these studies was to find a way to delay the absorption and increase the efficacy in treating spastic colon and irritable bowel syndrome. Menthol is fat-soluble and therefore is rapidly absorbed from the proximal small intestine when taken orally [116]. This makes it particularly useful for targeting disorders of the intestine. One of the first studies to look at peppermint oil ingestion was performed in 1984 [117] in which a comparison was done between the oral administration of gelatine capsules containing peppermint oil versus a Colpermin preparation (Tillotts Laboratories), a commercial peppermint preparation. The comparison was done in six healthy volunteers and repeated in six ileostomy subjects. The authors measured the levels of menthol in urine and found that Colpermin led to a more delayed-release of menthol compared to a gelatine capsule [117]. A second study conducted 3 years later, compared the Colpermin preparation with another new capsule administration (Mintec SK\&F Ltd.). The authors affirmed that the Colpermin preparation delivered menthol more effectively to the distal small intestine and ascending colon than the Mintec formulation [116]. In 1990, Kaffenberger [118] studied the administration of $180 \mathrm{mg}$ of peppermint oil in an enteric-coated capsule, identifying menthol glucuronide in urine by GC-FID [118].

In another study focusing on the kinetics of menthol metabolism [119], the authors used ${ }^{13} \mathrm{C}$ glucose administered with peppermint oil to detect ${ }^{13} \mathrm{C}$-menthol glucuronide. In a later study, the same authors used deuterated water administered with peppermint to detect menthol glucuronide by ${ }^{2} \mathrm{H}-\mathrm{NMR}$ spectroscopy [120]. Finally, the most recent study analyzed urine and plasma by GC-MS after intragastric administration of peppermint oil [121]. Approximately, 70\% of the administered menthol and its metabolites were excreted in the urine, and this amount fluctuated independent of the dose. The main metabolite identified in plasma and urine was menthol glucuronide along with lesser amounts of mono-hydroxylated menthol glucuronic acid and di-hydroxylated menthol glucuronic acid.

While menthol is highly abundant in peppermint (40\%), it can also be found in sunflower petals (essential oils), tarragon (0.1\%) [122], basil, and thyme (traces). However, due to the high menthol content in peppermint compared to the other two herbs and given that sunflower petals are not an edible food, menthol can be considered a specific marker of peppermint. Given that menthol glucuronide is also detectable in urine, this compound could also be a suitable biomarker of peppermint intake, particularly after capsular oral administration. However, further analysis in peppermint administration is needed for a suitable evaluation of menthol as potential food intake biomarker. In the case of spearmint, because no studies have yet been performed for spearmint BFIs, it is clear that spearmint interventions studies need to be undertaken.

\section{Saffron}

Saffron is among the world's most costly spices. It comes from the dried flower stigma of Crocus sativus. This is a flower that is native to Southwest Asia but which is now cultivated in Greece, India, Iran, Morocco, and Spain. Saffron is mainly used as a seasoning and coloring agent in food, particularly in Persian, Indian, European, and Arab cuisines. Saffron contains more than 150 volatile 
and aroma-yielding compounds. Safranal (2,6,6-trimethyl-1,3-cyclohexadiene-1-carboxaldehyde) is the major compound $(70 \%)$ in the volatile fraction of saffron [123, 124]. Saffron also has a number of non-volatile active components many of which are carotenoids, including zeaxanthin, lycopene, and various $\alpha-$ and $\beta$-carotenes. However, the golden yellow-orange color of saffron is primarily the result of the carotenoid $\alpha$-crocin, a glycosyl ester of crocetin. Picrocrocin (4-( $\beta$-d-glucopyranosyloxy)-2,6,6-trimethyl-1-cyclohexene-1-carboxaldehyde) has also been found in saffron spice from 0.8 to $26.6 \%$ on a dry basis. This compound is responsible for saffron's bitter taste [124]. In addition, saffron contains two important vitamins: riboflavin and thiamine. Riboflavin values range from 56 to $138 \mu \mathrm{g} / \mathrm{g}$, and are the highest reported for any food. Thiamine values range from 0.7 to $4 \mu \mathrm{g} / \mathrm{g}$, which are well within the range of values reported in many vegetables [125]. Saffron extracts and tinctures have been used as antispasmodic agents, gingival sedatives, nerve sedatives, expectorants, stimulants, and aphrodisiacs.

Only one study has explored BFIs for saffron [126]. This was a single dose study that looked at saffron products in the plasma of four healthy volunteers using solid phase extraction-high-performance liquid chromatography (SPE-HPLC). Saffron was administered in the form of saffron tea [126]. Crocetin was tracked from the plasma of subjects and was found to be rapidly absorbed, being detected in systemic circulation after $2 \mathrm{~h}$ of administration, with the compound still being present for up to $24 \mathrm{~h}$. A separate pharmacokinetic study that used purified crocetin extracted from Gardenia jasminoides fruit showed a strong dose-dependent absorption profile into the bloodstream of 10 healthy adult subjects [127].

While crocetin is also present in Gardenia jasminoides, this particular flower is not considered a common edible food so, in this regard, crocetin could be considered a specific BFI of saffron in plasma. The analytical performance of this biomarker has been well documented. However, additional studies are needed with regard to cumulative aspects (robustness in complex meals). Likewise, a comparison with other markers for the same food could certainly help with validating this BFI.

\section{Sage}

Sage or Salvia officinalis is a medicinal plant belonging to the Lamiaceae family. It is an aromatic herb native to the Mediterranean region but now widely distributed throughout the world. Sage has been used in traditional medicine for the treatment of seizures, ulcers, gout, rheumatism, inflammation, dizziness, tremors, paralysis, diarrhea, and hyperglycemia [128]. Sage has a savory, slightly peppery flavor. It is strongly aromatic, and is characterized by a medicinal, lemony, or bitter taste. It is used for seasoning and flavoring in many different foods including sausages and stuffing. The major components present in sage are $\alpha$-thujone (11.55-19.23\%), viridiflorol (9.94-19.46\%), 1,8-cineole (8.85-15.60\%), camphor (5.08-15.06\%), manool (5.52-13.06\%), $\beta$-caryophyllene (2.63-9.24\%), $\alpha$-humulene (1.93-8.94\%), and $\beta$-thujone $(5.45-6.17 \%)$ [129]. Some of the major phenolic compounds found in sage are rosmarinic acid, caffeic acid, carnosol, and carnosic acid. All of these polyphenols are also present in rosemary, thyme, or oregano as previously discussed.

Only one BFI study has been performed on sage in humans [130]. In this acute study, a single female volunteer was involved in a pharmacokinetic evaluation of 1,8-cineole after ingestion of sage tea [130]. The compounds identified in plasma and urine by GC-MS and LC-MS were 1,8-cineole and a number of its derivatives (2-hydroxy-1,8-cineole, 3-hydroxy-1,8-cineole, 7-hydroxy -1,8-cineole, 9-hydroxy-1,8-cineole). 1,8-cineole is also known as eucalyptol, and is a major component of essential oils from Eucalyptus polybractea. This monoterpene is present in numerous spices, such as rosemary, sage, basil, and laurel. It is also used in pharmaceutical preparations to treat coughs, muscular pain, neurosis, rheumatism, asthma, and urinary stones [131].

As mentioned earlier, other compounds present in rosemary and thyme are also present in sage. Interestingly, there are no intervention studies reporting any of these compounds after sage intake. While 1,8-cineole is detectable in urine after sage intake, it is important to note that 1,8-cineole is found in several other herbs belonging to the Laminacea family. Therefore, 1,8-cineole and its derivates cannot be considered to be specific for sage intake. Furthermore, its rapid metabolism makes it a poor BFI for most members of the Laminacea family. We conclude that there are no useful BFIs for sage intake that have yet been discovered or reported in the literature.

\section{Turmeric (curcumin)}

Turmeric is a rhizomatous herbaceous perennial plant (Curcuma longa) belonging to the ginger family, Zingiberaceae. It is native to Southeast Asia. Turmeric is a key ingredient in many Asian dishes and is used mainly as a coloring agent. The most notable phytochemical components of turmeric root include compounds called curcuminoids, such as curcumin (diferuloylmethane), demethoxycurcumin (DMC), and bisdemethoxycurcumin (BDMC). Curcumin is a polyphenolic molecule that constitutes $3.14 \%$ (on average) of powdered turmeric Curcumin is what gives the spice its yellow color [132]. The rhizome oils of turmeric contain more than 40 identifiable compounds, with the major constituents being 
$\alpha$-turmerone (30-32\%), aromatic-turmerone (17-26\%), and $\beta$-turmerone (15-18\%) [133].

Curcumin is a particularly well-studied spice. A total of 18 research papers were found for this review just for curcumin. This interest is likely due to the multiple biological or health activities attributed to it, including antioxidant, anti-inflammatory, and anti-tumor activities [134]. Recent clinical studies with curcumin have demonstrated additional health benefits relating to treating immune deficiencies, improving cardiovascular health, treating depression [135], combating Alzheimer's disease, treating diabetes [136], arthritis, and inflammatory bowel disease [137, 138]. Curcumin has very low oral bioavailability, which is primarily due to its poor solubility, low absorption, rapid metabolism, and systemic elimination. As a result, most ingested curcumin is excreted through the feces unmetabolized. The small portion that is absorbed is extensively converted to its water-soluble metabolites, glucuronides, and sulfates. These metabolites include curcumin-O-glucuronide (COG), curcumin-O-sulfate (COS) [25, 138], [139], [6, 140, 141], and tetrahydrocurcumin (THC) [138, 142, 143]. Apart from glucuronidation and sulfation, other biotransformations could occur, which yield other unspecific metabolites such as ferulic acid and vanillic acid [132]. COG, the major curcumin metabolite, is relatively hydrophilic compared to curcumin due to its conjugation to glucuronic acid [141].

Several pharmacokinetic studies with healthy volunteers $[139,144,145]$ and colorectal cancer patients [6] revealed that curcumin has a plasma $T_{\max }$ between 1 and $4 \mathrm{~h}$, with conjugates persisting for up to $36 \mathrm{~h}$ after $10 \mathrm{~g}$ of curcumin intake [139]. Sharma et al. [145] determined that there was no detectable curcumin or any of its metabolites in the blood or urine of patients after the administration of $440-2200 \mathrm{mg}$ of "curcuma" extract per day (containing $36-180 \mathrm{mg}$ of curcumin) in advanced colorectal cancer patients. Another study performed by Cheng et al. [144] demonstrated that the peak concentrations of curcumin in serum after administration of 4,6 , and $8 \mathrm{~g}$ of curcumin (given in the form of tablets) were $0.51,0.64$, and $1.77 \mu \mathrm{M}$, respectively. However, doses below $4 \mathrm{~g}$ were barely detectable. Lao et al. [146] could not detect curcumin in the serum of volunteers with doses ranging from 0.5 to $8.0 \mathrm{~g}$ of curcumin.

Due to curcumin's poor absorption, poor bioavailability, and fast excretion, several studies have explored different vehicles for curcumin administration to increase its bioavailability. Oral administration of curcumin in " $\mathrm{C} 3 \mathrm{com}$ plex," which consists of $450 \mathrm{mg}$ of curcumin, $30 \mathrm{mg}$ of demethoxycurcumin (DMC), and $20 \mathrm{mg}$ of bisdemethoxycurcumin (BDMC) [6] was extensively tested by several research groups [6, 139, 141, 143, 145-147]. Nearly all groups were able to detect COG up to $24 \mathrm{~h}$ after oral administration of $4 \mathrm{~g}$ of curcumin. However, no curcumin could be detected for the same subjects after the same time period [141]. This suggests that curcumin-O-glucuronide (COG) has a much higher half-life and is more suitable as a curcumin/turmeric BFI.

Another interesting study of curcumin bioavailability has been conducted by Jager et al. [138]. These researchers studied the absorption of formulated curcumin versus unformulated curcumin. Their formulated curcumin used a combination of a hydrophilic carrier, cellulosic derivatives, and natural antioxidants. This formulation was found to significantly increase curcuminoid levels in the blood in comparison to the unformulated product [138]. Theracurmin, a colloidal nanoparticle dispersion of curcumin, is reported to have a much higher bioavailability (27-fold higher) than any other available preparations [148]. A recent pharmacokinetic study conducted in both mice and humans [149], looked at fresh turmeric-derived curcuminoids versus dry turmeric-derived curcuminoids. These researchers showed that fresh curcuminoids permitted better plasma delivery and better absorption. Baum and co-workers suggested that curcumin consumed with food appears to accelerate its absorption [132].

Almost all of turmeric/curcumin intake studies that we found were performed in blood (plasma or serum). A smaller number of studies looked at other biofluids or excreta such as urine and feces. In almost all cases, curcumin was barely detected. Moderately suitable biomarkers of curcumin consumption were reported in plasma after the intake of formulated curcumin, such as BDMC, DMC, THC, COG, and COS, as opposed to unformulated administration. However, in feces and colon tissue, only curcumin itself could be detected. In conclusion, formulated curcumin, curcumin biotransformation products, and curcuminoids can be detected in plasma and in feces, but due to the fact that curcumin was administered in a formulated preparation, these biomarkers cannot be considered robust BFIs of turmeric/curcumin consumption.

\section{Conclusion}

As far as we are aware, this is the first comprehensive review of biomarkers of food intake for common herbs and spices. We believe it provides a useful overview of what is known, how most herb and spice BFI studies are done, and what needs to be done to improve the current state of knowledge of herb and spice BFIs. Based on our analysis, we found that most BFI studies of herbs and spices were typically performed through the administration of capsules, tablets or other "artificial" forms. In many cases, these studies administered a particularly abundant, carefully purified chemical component of the herb or spice, not in the form of the whole food. Technically, this means that this particular compound or family of compounds was/were evaluated 
in a supervised manner, not in an untargeted manner using the whole food (spice or herb) of interest. This suggests that potentially synergistic or antagonistic factors concerning the consumption of the herb or spice along with possible matrix effects in terms of absorption and bioavailability were not typically evaluated in these studies. Given that herbs and spices are normally consumed in combination with other foods, the influence of other food constituents taken in the same meal was not particularly well addressed in any of the studies that we reviewed. Because our focus was on human metabolism and biomarkers of human consumption, we restricted our reviews to human intervention trials. For the most part, animal models were not considered, except for a few specific cases where interesting or compelling biomarkers was reported. Overall, we were surprised by how little work has been done on BFIs in herbs and spices. From an initial list of 25 herbs and spices, we found that only 6 had useful or sufficiently robust BFI studies, and in many cases only a single study was completed. Relatively few fully validated BFIs were identified, although several promising or putative BFIs were described, and these will likely be confirmed if further validation studies are completed. Based on our data, it is clear that further research needs to be performed in the evaluation of herbs and spices in human intervention studies.

\section{Additional file}

Additional file 1: Tables S1. and Table S2. describing the literature

search criteria for herbs and spices. (DOCX $17 \mathrm{~kb}$ )

\section{Acknowledgements}

Not applicable.

\section{Funding}

FoodBAll is a project funded by the BioNH call (grant number 529051002) under the Joint Programming Initiative, "A Healthy Diet for a Healthy Life." The project is funded nationally by the respective Research Councils including a grant from the Canadian Institutes of Health Research (CIHR) to DSW.

\section{Availability of data and materials}

Not applicable.

\section{Authors' contributions}

The search of literature was performed by RVF. The articles were reviewed and selected by ARR, TS, and RVF. The preparation of the tables was done by TOO, NW, and RVF. The first draft was prepared by RVF and revised by DSW. The final manuscript following several revisions by all co-authors was finally accepted by all of them.

\section{Ethics approval and consent to participate}

Not applicable.

\section{Consent for publication}

Not applicable.

\section{Competing interests}

The authors declare that they have no competing interests.

\section{Publisher's Note}

Springer Nature remains neutral with regard to jurisdictional claims in published maps and institutional affiliations.

\section{Author details}

${ }^{1}$ Department of Biological Sciences, University of Alberta, Edmonton, AB T6G 2E9, Canada. ${ }^{2}$ Department of Computing Science, University of Alberta, Edmonton, AB T6G 2E8, Canada.

Received: 9 November 2018 Accepted: 9 April 2019

Published online: 22 May 2019

\section{References}

1. Embuscado ME. Spices and herbs: Natural sources of antioxidants-a mini review. J Funct Foods. 2015;18 Part B:811-9.

2. Codex Alimentarius Comission. FAO/WHO Food Standards Programme. Code of Hygienic Practices for Spices and Dried Aromatic Plants. 1995. Rev2014. http://www.fao.org/input/download/standards/27/CXP_042e_2014. pdf. Accessed May 2016.

3. Peter KV. Handbook of herbs and spices: CRC Press; 2004. https://www. elsevier.com/books/handbook-of-herbs-andspices/peter/978-0-85709-039-3.

4. Rubio L, Motilva MJ, Romero MP. Recent advances in biologically active compounds in herbs and spices: a review of the most effective antioxidant and anti-inflammatory active principles. Crit Rev Food Sci Nutr. 2013;539: 943-53.

5. Aggarwal BB, Sung B. Pharmacological basis for the role of curcumin in chronic diseases: an age-old spice with modern targets. Trends Pharmacol Sci. 2009:302:85-94.

6. Garcea G, Berry DP, Jones DJ, Singh R, Dennison AR, Farmer PB, Sharma RA, Steward WP, Gescher AJ. Consumption of the putative chemopreventive agent curcumin by cancer patients: assessment of curcumin levels in the colorectum and their pharmacodynamic consequences. Cancer Epidemiol Biomark Prev. 2005;141:120-5.

7. Lai PK, Roy J. Antimicrobial and chemopreventive properties of herbs and spices. Curr Med Chem. 2004;1111:1451-60.

8. Mashmoul M, Azlan A, Khaza'ai H, Yusof B, Noor S. Saffron: a natural potent antioxidant as a promising anti-obesity drug. Antioxidants. 2013;24:293.

9. Bi X, Lim J, Henry CJ. Spices in the management of diabetes mellitus. Food Chem. 2017;217:281-93.

10. Tapsell LC, Hemphill I, Cobiac L, Patch CS, Sullivan DR, Fenech M, Roodenrys S, Keogh JB, Clifton PM, Williams PG, et al. Health benefits of herbs and spices: the past, the present, the future. Med J Aust. 2006;1854(Suppl):S4-24.

11. Satheeshkumar N, Vijayan RSK, Lingesh A, Santhikumar S, Vishnuvardhan C. Spices: Potential Therapeutics for Alzheimer's Disease. In: Essa MM, Akbar M, Guillemin G, editors. The Benefits of Natural Products for Neurodegenerative Diseases. Cham: Springer International Publishing; 2016. p. 57-78.

12. Akhondzadeh $\mathrm{S}$, Noroozian M, Mohammadi M, Ohadinia S, Jamshidi AH, Khani M. Salvia officinalis extract in the treatment of patients with mild to moderate Alzheimer's disease: a double blind, randomized and placebocontrolled trial. J Clin Pharm Ther. 2003;281:53-9.

13. Rastogi S, Pandey MM, Rawat A. Spices: Therapeutic Potential In Cardiovascular Health. Curr Pharm Des. 2017;23(7):989-98.

14. Sun F, Xiong S, Zhu Z. Dietary capsaicin protects cardiometabolic organs from dysfunction. Nutrients. 2016;85:174.

15. Shahidi F, Ambigaipalan P. Phenolics and polyphenolics in foods, beverages and spices: antioxidant activity and health effects-a review. J Funct Foods. 2015;18 Part B:820-97.

16. Anderson CA, Cobb LK, Miller ER, Woodward M, Hottenstein A, Chang AR, Mongraw-Chaffin M, White K, Charleston J, Tanaka T, et al. Effects of a behavioral intervention that emphasizes spices and herbs on adherence to recommended sodium intake: results of the SPICE randomized clinical trial. Am J Clin Nutr. 2015: 1023:671-9.

17. Opara El, Chohan M. Culinary herbs and spices: their bioactive properties, the contribution of polyphenols and the challenges in deducing their true health benefits. Int J Mol Sci. 2014;1510:19183-202.

18. Cowan MM. Plant products as antimicrobial agents. Clin Microbiol Rev. 1999;124:564-82.

19. Calucci L, Pinzino C, Zandomeneghi M, Capocchi A, Ghiringhelli S, Saviozz F, Tozzi S, Galleschi L. Effects of gamma-irradiation on the free radical and antioxidant contents in nine aromatic herbs and spices. J Agric Food Chem. 2003:514:927-34. 
20. Burt S. Essential oils: their antibacterial properties and potential applications in foods--a review. Int J Food Microbiol. 2004;943:223-53.

21. Fabio A, Corona A, Forte E, Quaglio P. Inhibitory activity of spices and essential oils on psychrotrophic bacteria. New Microbiol. 2003;261:115-20.

22. Kalemba D, Kunicka A. Antibacterial and antifungal properties of essential oils. Curr Med Chem. 2003;1010:813-29.

23. WHO: GEMS/Food consumption Database. https://www.who.int/nutrition/ landscape_analysis/nlis_gem_food/en/. Accessed September 2016.

24. WHO. GEMS/Food regional diets : regional per capita consumption of raw and semi-processed agricultural commodities / prepared by the Global Environment Monitoring System/Food Contamination Monitoring and Assessment Programme (GEMS/Food). 2003.

25. Sharma RA, McLelland HR, Hill KA, Ireson CR, Euden SA, Manson MM, Pirmohamed M, Marnett LJ, Gescher AJ, Steward WP. Pharmacodynamic and pharmacokinetic study of oral Curcuma extract in patients with colorectal cancer. Clin Cancer Res. 2001;77:1894-900.

26. CBI Ministry of foreign Affairs. Spices and herbs: a survey of the Netherlands and other major markets in the European Union, centre for promotion of imports from developing countries. 1999. http://uww.faoda.org/download/Spices_and_Herbs_ Survey.pdf.

27. Williams PG. Health benefits of herbs and spices. Publ Health Med J Aust. 2006;1854:Supplement S17-8.

28. Dragsted LO, Gao Q, Praticò G, Manach C, Wishart DS, Scalbert A, Feskens EJM. Dietary and health biomarkers — time for an update. Genes Nutr. 2017;12:24.

29. Praticò G, Gao Q, Scalbert A, Vergères $G$, Kolehmainen M, Manach C, Brennan L, Pedapati SH, Afman LA, Wishart DS, et al. Guidelines for biomarker of food intake reviews (BFIRev): how to conduct an extensive literature search for biomarker of food intake discovery. Genes Nutr. 2018;131:3.

30. EFSA. Application of systematic review methodology to food and feed safety assessments to support decision making. Guidance for those carrying out systematic reviews European Food Safety Authority. EFSA J. 2010;86:1637.

31. Higgins JPT, Green S. Cochrane handbook for systematic reviews of interventions version 5.1.0 [updated March 2011]. 5th ed. London: The Cochrane Collaboration. p. 2011.

32. Moher D, Liberati A, Tetzlaff J, Altman DG, Group P. Preferred reporting items for systematic reviews and meta-analyses: the PRISMA statement. Ann Intern Med. 2009;1514:264-9 W64.

33. Dragsted LO, Gao Q, Scalbert A, Vergères G, Kolehmainen M, Manach C, Brennan L, Afman LA, Wishart DS, Andres Lacueva C, et al. Validation of biomarkers of food intake — critical assessment of candidate biomarkers. Genes Nutr. 2018;13:14.

34. Marinov V, Valcheva-Kuzmanova S. Review on the pharmacological activities of anethole. Scr Sci Pharm. 2015;22:14-9.

35. Rodrigues VM, Rosa PT, Marques MO, Petenate AJ, Meireles MA. Supercritical extraction of essential oil from aniseed (Pimpinella anisum L) using $\mathrm{CO} 2$ : solubility, kinetics, and composition data. J Agric Food Chem. 2003;516:1518-23.

36. Caldwell J, Sutton JD. Influence of dose size on the disposition of trans-[methoxy14Clanethole in human volunteers. Food Chem Toxicol. 1988:262:87-91.

37. Schulz K, Schlenz K, Metasch R, Malt S, Romhild W, Dressler J. Determination of anethole in serum samples by headspace solid-phase microextractiongas chromatography-mass spectrometry for congener analysis. J Chromatogr A. 2008;12002:235-41.

38. Barfi A, Nazem H, Saeidi I, Peyrovi M, Afsharzadeh M, Barfi B, Salavati H. Insyringe reversed dispersive liquid-liquid microextraction for the evaluation of three important bioactive compounds of basil, tarragon and fennel in human plasma and urine samples. J Pharm Biomed Anal. 2016;121:123-34.

39. Kobata K, Todo T, Yazawa S, Iwai K, Watanabe T. Novel Capsaicinoid-like substances, Capsiate and Dihydrocapsiate, from the fruits of a nonpungent cultivar, CH-19 sweet, of pepper (Capsicum annuum L.). J Agric Food Chem. 1998:465:1695-7

40. Chaiyata P, Puttadechakum S, Komindr S. Effect of chili pepper (Capsicum frutescens) ingestion on plasma glucose response and metabolic rate in Thai women. J Med Assoc Thail. 2003;869:854-60.

41. Chaiyasit K, Khovidhunkit W, Wittayalertpanya S. Pharmacokinetic and the effect of capsaicin in Capsicum frutescens on decreasing plasma glucose level. J Med Assoc Thail. 2009;921:108-13.

42. Nishino A, Ichihara T, Takaha T, Kuriki T, Nihei H, Kawamoto K, Yasui H, Maoka T. Accumulation of paprika carotenoids in human plasma and erythrocytes. J Oleo Sci. 2015;6410:1135-42.

43. Nishino A, Yasui H, Maoka T. Reaction of paprika carotenoids, Capsanthin and Capsorubin, with reactive oxygen species. J Agric Food Chem. 2016; 6423:4786-92
44. Kim J-S, Lee W-M, Rhee HC, Kim S. Red paprika (Capsicum annuum L.) and its main carotenoids, capsanthin and $\beta$-carotene, prevent hydrogen peroxide-induced inhibition of gap-junction intercellular communication. Chem Biol Interact. 2016;254:146-55.

45. Parry JW. The story of spices. Econ Bot. 1955;92:190-207.

46. Khan A, Safdar M, Ali Khan MM, Khattak KN, Anderson RA. Cinnamon improves glucose and lipids of people with type 2 diabetes. Diabetes Care. 2003;2612:3215-8

47. Jayaprakasha GK, Rao L. Chemistry, biogenesis, and biological activities of Cinnamomum zeylanicum. Crit Rev Food Sci Nutr. 2011;516:547-62.

48. Abraham K, Pfister M, Wohrlin F, Lampen A. Relative bioavailability of coumarin from cinnamon and cinnamon-containing foods compared to isolated coumarin: a four-way crossover study in human volunteers. Mol Nutr Food Res. 2011;554:644-53.

49. Yuan J, Bucher JR, Goehl TJ, Dieter MP, Jameson CW. Quantitation of cinnamaldehyde and cinnamic acid in blood by HPLC. J Anal Toxicol. 1992;166: 359-62.

50. Pelkonen O, Rautio A, Raunio H, Pasanen M. CYP2A6: a human coumarin 7hydroxylase. Toxicology. 2000;1441-3:139-47.

51. Rautio A, Kraul H, Kojo A, Salmela E, Pelkonen O. Interindividual variability of coumarin 7-hydroxylation in healthy volunteers. Pharmacogenetics. 1992;25:227-33.

52. Lake BG. Coumarin metabolism, toxicity and carcinogenicity: relevance for human risk assessment. Food Chem Toxicol. 1999;374:423-53.

53. Blahová J, Svobodová Z. Assessment of Coumarin levels in ground cinnamon available in the Czech retail market. ScientificWorldJournal. 2012; 2012:263851.

54. Ballin NZ, Sørensen AT. Coumarin content in cinnamon containing food products on the Danish market. Food Control. 2014;38:198-203.

55. Simon JE, M.R. Morales, W.B. Phippen, R.F. Vieira, and Z. Hao. Basil: A Source of Aroma Compounds and a Popular Culinaryand Ornamental Herb. 1999. https://www.hort.purdue.edu/newcrop/proceedings1999/v4-499.html. Accessed June 2016

56. Hussain Al, Anwar F, Hussain Sherazi ST, Przybylski R. Chemical composition, antioxidant and antimicrobial activities of basil (Ocimum basilicum) essential oils depends on seasonal variations. Food Chem. 2008;1083:986-95.

57. Wannissorn B, Jarikasem S, Siriwangchai T, Thubthimthed S. Antibacterial properties of essential oils from Thai medicinal plants. Fitoterapia. 2005;762:233-6.

58. Joshi RK. Chemical composition and antimicrobial activity of the essential oil of Ocimum basilicum L. (sweet basil) from Western Ghats of north West Karnataka, India. Anc Sci Life. 2014;333:151-6.

59. Politeo O, Jukic M, Milos M. Chemical composition and antioxidant capacity of free volatile aglycones from basil (Ocimum basilicum L.) compared with its essential oil. Food Chem. 2007:1011:379-85.

60. Aglarova AM, Zilfikarov IN, Severtseva OV. Biological characteristics and useful properties of tarragon (Artemisia dracunculus L.) (review). Pharm Chem J. 2008;422:81-6.

61. Obolskiy D, Pischel I, Feistel B, Glotov N, Heinrich M. Artemisia dracunculus L. (tarragon): a critical review of its traditional use, chemical composition, pharmacology, and safety. J Agric Food Chem. 2011;5921:11367-84.

62. Sayyah M, Nadjafnia L, Kamalinejad M. Anticonvulsant activity and chemical composition of Artemisia dracunculus L. essential oil. J Ethnopharmacol. 2004;942:283-7

63. Zeller A, Horst K, Rychlik M. Study of the metabolism of estragole in humans consuming fennel tea. Chem Res Toxicol. 2009;2212:1929-37.

64. Surh Y-J. Anti-tumor promoting potential of selected spice ingredients with antioxidative and anti-inflammatory activities: a short review. Food Chem Toxicol. 2002:408:1091-7.

65. Mascolo N, Jain R, Jain SC, Capasso F. Ethnopharmacologic investigation of ginger (Zingiber officinale). J Ethnopharmacol. 1989;271-2:129-40.

66. Park M, Bae J, Lee DS. Antibacterial activity of [10]-gingerol and [12]gingerol isolated from ginger rhizome against periodontal bacteria. Phytother Res. 2008;2211:1446-9.

67. Rahmani AH, FMA S, Aly SM. Active ingredients of ginger as potential candidates in the prevention and treatment of diseases via modulation of biological activities. Int J Physiol Pathophysiol Pharmacol. 2014;62:125-36.

68. Govindarajan VS. Ginger--chemistry, technology, and quality evaluation: part 1. Crit Rev Food Sci Nutr. 1982;171:1-96.

69. Zick SM, Djuric Z, Ruffin MT, Litzinger AJ, Normolle DP, Alrawi S, Feng MR, Brenner DE. Pharmacokinetics of 6-gingerol, 8-gingerol, 10-gingerol, and 6shogaol and conjugate metabolites in healthy human subjects. Cancer Epidemiol Biomark Prev. 2008;178:1930-6. 
70. Yu Y, Zick S, Li X, Zou P, Wright B, Sun D. Examination of the pharmacokinetics of active ingredients of ginger in humans. AAPS J. 2011;133:417-26.

71. Chen H, Soroka DN, Hu Y, Chen X, Sang S. Characterization of thiolconjugated metabolites of ginger components shogaols in mouse and human urine and modulation of the glutathione levels in cancer cells by [6]-shogaol. Mol Nutr Food Res. 2013;573:447-58.

72. Muchtaridi SA, Apriyantono A, Mustarichie R. Identification of compounds in the essential oil of nutmeg seeds (Myristica fragrans Houtt.) that inhibit locomotor activity in mice. Int J Mol Sci. 2010;1111:4771-81.

73. Beyer J, Ehlers D, Maurer HH. Abuse of nutmeg (Myristica fragrans Houtt.): studies on the metabolism and the toxicologic detection of its ingredients elemicin, myristicin, and safrole in rat and human urine using gas chromatography/mass spectrometry. Ther Drug Monit. 2006;284:568-75.

74. Hallstrom H, Thuvander A. Toxicological evaluation of myristicin. Nat Toxins. 1997:55:186-92

75. Zheng GQ, Kenney PM, Zhang J, Lam LK. Inhibition of benzo[a]pyreneinduced tumorigenesis by myristicin, a volatile aroma constituent of parsley leaf oil. Carcinogenesis. 1992;1310:1921-3.

76. Ahmad H, Tijerina MT, Tobola AS. Preferential overexpression of a class MU glutathione S-transferase subunit in mouse liver by myristicin. Biochem Biophys Res Commun. 1997;2363:825-8.

77. Rossi P-G, Bao L, Luciani A, Panighi J, Desjobert J-M, Costa J, Casanova J, Bolla J-M, Berti L. (E)-Methylisoeugenol and Elemicin: antibacterial components of Daucus carota L. essential oil against campylobacter jejuni. J Agric Food Chem. 2007;5518:7332-6.

78. MacLeod AJ, Snyder CH, Subramanian G. Volatile aroma constituents of parsley leaves. Phytochemistry. 1985;2411:2623-7.

79. De Vincenzi M, De Vincenzi A, Silano M. Constituents of aromatic plants: elemicin. Fitoterapia. 2004;756:615-8.

80. Heikes DL. SFE with GC and MS determination of safrole and related allylbenzenes in sassafras teas. J Chromatogr Sci. 1994;327:253-8.

81. Parthasarathy VA, Chempakam B, Zachariah TJ. Chemistry of Spices: CABI Oxfordshire; 2008. https:/catbull.com/alamut/Bibliothek/Chemistry_of_Spices.pdf.

82. Mockute D, Bernotiene G, Judzentiene A. The essential oil of Origanum vulgare L. ssp. vulgare growing wild in Vilnius district (Lithuania). Phytochemistry. 2001;571:65-9.

83. Vallverdú-Queralt A, Regueiro J, Martínez-Huélamo M, Rinaldi Alvarenga JF, Leal LN, Lamuela-Raventos RM. A comprehensive study on the phenolic profile of widely used culinary herbs and spices: rosemary, thyme, oregano, cinnamon, cumin and bay. Food Chem. 2014;154:299-307.

84. Farag RS, Daw ZY, Hewedi FM, El-Baroty GSA. Antimicrobial activity of some Egyptian spice essential oils. J Food Prot. 1989;529:665-7.

85. Rota MC, Herrera A, Martínez RM, Sotomayor JA, Jordán MJ. Antimicrobial activity and chemical composition of Thymus vulgaris, Thymus zygis and Thymus hyemalis essential oils. Food Control. 2008;197:681-7.

86. Soković M, Glamočlija J, Marin PD, Brkić D, van Griensven LJ. Antibacterial Effects of the Essential Oils of Commonly Consumed Medicinal Herbs Using an In Vitro Model. Molecules. 2010;1511:7532.

87. Hudaib M, Speroni E, Di Pietra AM, Cavrini V. GC/MS evaluation of thyme (Thymus vulgaris L.) oil composition and variations during the vegetative cycle. J Pharm Biomed Anal. 2002;294:691-700.

88. Ibarra A, Cases J, Roller M, Chiralt-Boix A, Coussaert A, Ripoll C. Carnosic acid-rich rosemary (Rosmarinus officinalis L.) leaf extract limits weight gain and improves cholesterol levels and glycaemia in mice on a high-fat diet. $\mathrm{Br}$ J Nutr. 2011;1068:1182-9.

89. Kulisic T, Krisko A, Dragovic-Uzelac V, Milos M, Pifat G. The effects of essential oils and aqueous tea infusions of oregano (Origanum vulgare L. spp. hirtum), thyme (Thymus vulgaris L.) and wild thyme (Thymus serpyllum L.) on the copper-induced oxidation of human low-density lipoproteins. Int J Food Sci Nutr. 2007:582:87-93.

90. Prieto JM, lacopini $\mathrm{P}$, Cioni $\mathrm{P}$, Chericoni S. In vitro activity of the essential oils of Origanum vulgare, Satureja montana and their main constituents in peroxynitrite-induced oxidative processes. Food Chem. 2007;1043:889-95.

91. Pérez-Fons L, GarzÓn MT, Micol V. Relationship between the antioxidant capacity and effect of rosemary (Rosmarinus officinalis L.) polyphenols on membrane phospholipid order. J Agric Food Chem. 2010;581:161-71.

92. Mueller M, Hobiger S, Jungbauer A. Anti-inflammatory activity of extracts from fruits, herbs and spices. Food Chem. 2010;1224:987-96.

93. Lai C-S, Lee JH, Ho C-T, Liu CB, Wang J-M, Wang Y-J, Pan M-H. Rosmanol potently inhibits lipopolysaccharide-induced iNOS and COX-2 expression through downregulating MAPK, NF-KB, STAT3 and C/EBP signaling pathways. J Agric Food Chem. 2009;5722:10990-8.

94. Zeng HH, Tu PF, Zhou K, Wang H, Wang BH, Lu JF. Antioxidant properties of phenolic diterpenes from Rosmarinus officinalis. Acta Pharmacol Sin. 2001; 2212:1094-8.

95. Mechan AO, Fowler A, Seifert N, Rieger H, Wohrle T, Etheve S, Wyss A, Schuler G, Colletto B, Kilpert $\mathrm{C}$, et al. Monoamine reuptake inhibition and mood-enhancing potential of a specified oregano extract. Br J Nutr. 2011;1058:1150-63.

96. Mosele Jl, Martin-Pelaez S, Macia A, Farras M, Valls RM, Catalan U, Motilva MJ. Study of the catabolism of thyme phenols combining in vitro fermentation and human intervention. J Agric Food Chem. 2014;6245:10954-61.

97. Nurmi A, Nurmi T, Mursu J, Hiltunen R, Voutilainen S. Ingestion of oregano extract increases excretion of urinary phenolic metabolites in humans. $J$ Agric Food Chem. 2006;5418:6916-23.

98. Rubio L, Serra A, Macia A, Borras X, Romero MP, Motilva MJ. Validation of determination of plasma metabolites derived from thyme bioactive compounds by improved liquid chromatography coupled to tandem mass spectrometry. J Chromatogr B Analyt Technol Biomed Life Sci. 2012;905:75-84.

99. Rubió L, Farràs M, de La Torre R, Macià A, Romero M-P, Valls RM, Solà R, Farré M, Fitó M, Motilva M-J. Metabolite profiling of olive oil and thyme phenols after a sustained intake of two phenol-enriched olive oils by humans: Identification of compliance markers. Food Res Int. 2014;65 Part A:59-68.

100. Romo Vaquero M, Garcia Villalba R, Larrosa M, Yanez-Gascon MJ, Fromentin E, Flanagan J, Roller M, Tomas-Barberan FA, Espin JC, Garcia-Conesa MT. Bioavailability of the major bioactive diterpenoids in a rosemary extract: metabolic profile in the intestine, liver, plasma, and brain of Zucker rats. Mol Nutr Food Res. 2013;5710:1834-46.

101. Zhao Y, Sedighi R, Wang P, Chen H, Zhu Y, Sang S. Carnosic acid as the major bioactive component in rosemary extract ameliorates high-fat dietinduced obesity and metabolic syndrome in mice. J Agric Food Chem. 2015;6319:4843-52.

102. Meyer $\mathrm{H}$, Bolarinwa A, Wolfram G, Linseisen J. Bioavailability of apigenin from apiin-rich parsley in humans. Ann Nutr Metab. 2006;503:167-72.

103. Maher HM, Al-Zoman NZ, Al-Shehri MM, Al-Showiman H, Al-Taweel AM, Fawzy GA, Perveen S. Determination of Luteolin and Apigenin in herbs by capillary electrophoresis with diode Array detection. Instrum Sci Technol. 2015;436:611-25.

104. Pan MH, Lai CS, Ho CT. Anti-inflammatory activity of natural dietary flavonoids. Food Funct. 2010;11:15-31.

105. Shukla S, Gupta S. Apigenin: a promising molecule for cancer prevention. Pharm Res. 2010;276:962-78.

106. Zhang H, Chen F, Wang X, Yao H-Y. Evaluation of antioxidant activity of parsley (Petroselinum crispum) essential oil and identification of its antioxidant constituents. Food Res Int. 2006;398:833-9.

107. Simon JE, Quinn J. Characterization of essential oil of parsley. J Agric Food Chem. 1988:363:467-72.

108. Nielsen SE, Dragsted LO. Column-switching high-performance liquid chromatographic assay for determination of apigenin and acacetin in human urine with ultraviolet absorbance detection. J Chromatogr B Biomed Sci Appl. 1998;7132:379-86.

109. Nielsen SE, Young JF, Daneshvar B, Lauridsen ST, Knuthsen P, Sandstrom B, Dragsted LO. Effect of parsley (Petroselinum crispum) intake on urinary apigenin excretion, blood antioxidant enzymes and biomarkers for oxidative stress in human subjects. Br J Nutr. 1999;816:447-55.

110. Janssen K, Mensink RP, Cox FJ, Harryvan JL, Hovenier R, Hollman PC, Katan MB. Effects of the flavonoids quercetin and apigenin on hemostasis in healthy volunteers: results from an in vitro and a dietary supplement study. Am J Clin Nutr. 1998:672:255-62.

111. Charles DJ. Peppermint. In: Antioxidant properties of spices, herbs and other sources. New York: Springer New York; 2013. p. 469-75.

112. Kokkini S, Karousou R, Lanaras T. Essential oils of spearmint (Carvone-rich) plants from the island of Crete (Greece). Biochem Syst Ecol. 1995;234:425-30.

113. Schmidt E, Bail S, Buchbauer G, Stoilova I, Atanasova T, Stoyanova A, Krastanov A, Jirovetz L. Chemical composition, olfactory evaluation and antioxidant effects of essential oil from Mentha $x$ piperita. Nat Prod Commun. 2009:48:1107-12.

114. Işcan G, Kirimer N, Kürkcüoğlu M, Başer KH, Demirci F. Antimicrobial screening of Mentha piperita essential oils. J Agric Food Chem. 2002;5014:3943-6.

115. Eccles R. Menthol and related cooling compounds. J Pharm Pharmacol. 1994;468:618-30.

116. White DA, Thompson SP, Wilson CG, Bell GD. A pharmacokinetic comparison of two delayed-release peppermint oil preparations, Colpermin 
and Mintec, for treatment of the irritable bowel syndrome. Int J Pharm. 1987:401:151-5.

117. Somerville KW, Richmond CR, Bell GD. Delayed release peppermint oil capsules (Colpermin) for the spastic colon syndrome: a pharmacokinetic study. Br J Clin Pharmacol. 1984;184:638-40.

118. Kaffenberger RM, Doyle MJ. Determination of menthol and menthol glucuronide in human urine by gas chromatography using an enzymesensitive internal standard and flame ionization detection. J Chromatogr. 1990;5271:59-66.

119. Mendes AC, Caldeira MM, Silva C, Burgess SC, Merritt ME, Gomes F, Barosa C, Delgado TC, Franco F, Monteiro P, et al. Hepatic UDP-glucose 13C isotopomers from [U-13C]glucose: a simple analysis by $13 \mathrm{C} \mathrm{NMR}$ of urinary menthol glucuronide. Magn Reson Med. 2006:565:1121-5.

120. Ribeiro A, Caldeira MM, Carvalheiro M, Bastos M, Baptista C, Fagulha A, Barros $\mathrm{L}$, Barosa $\mathrm{C}$, Jones JG. Simple measurement of gluconeogenesis by direct $2 \mathrm{H}$ NMR analysis of menthol glucuronide enrichment from $2 \mathrm{H} 2 \mathrm{O}$. Magn Reson Med. 2005;542:429-34.

121. Hiki N, Kaminishi M, Hasunuma T, Nakamura M, Nomura S, Yahagi N, Tajiri $H$, Suzuki H. A phase I study evaluating tolerability, pharmacokinetics, and preliminary efficacy of L-menthol in upper gastrointestinal endoscopy. Clin Pharmacol Ther. 2011;902:221-8.

122. Verma MK, Anand R, Chisti AM, Kitchlu S, Chandra S, Shawl AS, Khajuria RK. Essential oil composition of Artemisia dracunculus L. (tarragon) growing in Kashmir -India. J Essent Oil Bear PI. 2010;133:331-5.

123. Maggi L, Sánchez AM, Carmona M, Kanakis CD, Anastasaki E, Tarantilis PA, Polissiou MG, Alonso GL. Rapid determination of safranal in the quality control of saffron spice (Crocus sativus L.). Food Chem. 2011;1271:369-73.

124. Sánchez AM, Carmona M, del Campo CP, Alonso GL. Solid-phase extraction for picrocrocin determination in the quality control of saffron spice (Crocus sativus L.). Food Chem. 2009;1 163:792-8.

125. Giaccio M. Crocetin from saffron: an active component of an ancient spice. Crit Rev Food Sci Nutr. 2004;443:155-72.

126. Chryssanthi DG, Lamari FN, Georgakopoulos CD, Cordopatis P. A new validated SPE-HPLC method for monitoring crocetin in human plasma-application after saffron tea consumption. J Pharm Biomed Anal. 2011:553:563-8.

127. Umigai N, Murakami K, Ulit MV, Antonio LS, Shirotori M, Morikawa $H$, Nakano T. The pharmacokinetic profile of crocetin in healthy adult human volunteers after a single oral administration. Phytomedicine. 2011;187:575-8.

128. Ghorbani A, Esmaeilizadeh M. Pharmacological properties of Salvia officinalis and its components. J Tradit Complement Med. 2017;74:433-40.

129. Ben Farhat M, Jordan MJ, Chaouech-Hamada R, Landoulsi A, Sotomayor JA. Variations in essential oil, phenolic compounds, and antioxidant activity of tunisian cultivated Salvia officinalis L. J Agric Food Chem. 2009:5721:10349-56.

130. Horst K, Rychlik M. Quantification of 1,8-cineole and of its metabolites in humans using stable isotope dilution assays. Mol Nutr Food Res. 2010;5410:1515-29.

131. Santos FA, Rao VS. Antiinflammatory and antinociceptive effects of 1,8cineole a terpenoid oxide present in many plant essential oils. Phytother Res. 2000;144:240-4

132. Baum L, Cheung SK, Mok VC, Lam LC, Leung VP, Hui E, Ng CC, Chow M, Ho PC, Lam S, et al. Curcumin effects on blood lipid profile in a 6-month human study. Pharmacol Res. 2007;566:509-14.

133. Sharma RK, Misra BP, Sarma TC, Bordoloi AK, Pathak MG, Leclerca PA. Essential oils of Curcuma longa L. from Bhutan. J Essent Oil Res. 1997;95:589-92.

134. Maheshwari RK, Singh AK, Gaddipati J, Srimal RC. Multiple biological activities of curcumin: a short review. Life Sci. 2006;7818:2081-7.

135. Seo H-J, Wang S-M, Han C, Lee S-J, Patkar AA, Masand PS, Pae C-U. Curcumin as a putative antidepressant. Expert Rev Neurother. 2015;153:269-80.

136. Zhang DW, Fu M, Gao SH, Liu JL. Curcumin and diabetes: a systematic review. Evid Based Complement Alternat Med. 2013;2013:636053.

137. Sreedhar R, Arumugam S, Thandavarayan RA, Karuppagounder V, Watanabe $\mathrm{K}$. Curcumin as a therapeutic agent in the chemoprevention of inflammatory bowel disease. Drug Discov Today. 2016;215:843-9.

138. Jager R, Lowery RP, Calvanese AV, Joy JM, Purpura M, Wilson JM. Comparative absorption of curcumin formulations. Nutr J. 2014;13:11.

139. Vareed SK, Kakarala M, Ruffin MT, Crowell JA, Normolle DP, Djuric Z, Brenner DE. Pharmacokinetics of curcumin conjugate metabolites in healthy human subjects. Cancer Epidemiol Biomark Prev. 2008;176:1411-7.

140. Dhillon N, Aggarwal BB, Newman RA, Wolff RA, Kunnumakkara AB, Abbruzzese $\mathrm{JL}, \mathrm{Ng}$ CS, Badmaev V, Kurzrock R. Phase II trial of curcumin in patients with advanced pancreatic cancer. Clin Cancer Res. 2008;1414:4491-9.
141. Chen W, Fan-Havard P, Yee LD, Cao Y, Stoner GD, Chan KK, Liu Z. A liquid chromatography-tandem mass spectrometric method for quantification of curcumin-O-glucuronide and curcumin in human plasma. J Chromatogr B Analyt Technol Biomed Life Sci. 2012;900:89-93.

142. Cao Y, Xu RX, Liu Z. A high-throughput quantification method of curcuminoids and curcumin metabolites in human plasma via highperformance liquid chromatography/tandem mass spectrometry. J Chromatogr B Analyt Technol Biomed Life Sci. 2014;949-950:70-8.

143. Garcea G, Jones DJ, Singh R, Dennison AR, Farmer PB, Sharma RA, Steward WP, Gescher AJ, Berry DP. Detection of curcumin and its metabolites in hepatic tissue and portal blood of patients following oral administration. $\mathrm{Br}$ J Cancer. 2004;905:1011-5.

144. Cheng AL, Hsu CH, Lin JK, Hsu MM, Ho YF, Shen TS, Ko JY, Lin JT, Lin BR, Ming-Shiang W, et al. Phase I clinical trial of curcumin, a chemopreventive agent, in patients with high-risk or pre-malignant lesions. Anticancer Res. 2001;214B:2895-900

145. Sharma RA, Euden SA, Platton SL, Cooke DN, Shafayat A, Hewitt HR, Marczylo TH, Morgan B, Hemingway D, Plummer SM, et al. Phase I clinical trial of oral curcumin: biomarkers of systemic activity and compliance. Clin Cancer Res. 2004;1020:6847-54.

146. Lao CD, Ruffin MT, Normolle D, Heath DD, Murray SI, Bailey JM, Boggs ME, Crowell J, Rock CL, Brenner DE. Dose escalation of a curcuminoid formulation. BMC Complement Altern Med. 2006;6:10.

147. Irving GR, Howells LM, Sale S, Kralj-Hans I, Atkin WS, Clark SK, Britton RG, Jones DJ, Scott EN, Berry DP, et al. Prolonged biologically active colonic tissue levels of curcumin achieved after oral administration--a clinical pilot study including assessment of patient acceptability. Cancer Prev Res (Phila). 2013;62:119-28.

148. Sasaki H, Sunagawa Y, Takahashi K, Imaizumi A, Fukuda H, Hashimoto T, Wada H, Katanasaka Y, Kakeya H, Fujita M, et al. Innovative preparation of curcumin for improved oral bioavailability. Biol Pharm Bull. 2011;345:660-5.

149. Krishnakumar IM, Kumar D, Ninan E, Kuttan R, Maliakel B. Enhanced absorption and pharmacokinetics of fresh turmeric (Curcuma longa $L$ ) derived curcuminoids in comparison with the standard curcumin from dried rhizomes. J Funct Foods. 2015;17:55-65.

150. Bernard BK, Tsubuku S, Kayahara T, Maeda K, Hamada M, Nakamura T, Shirai Y, Nakayama A, Ueno S, Mihara R. Studies of the toxicological potential of capsinoids: X. Safety assessment and pharmacokinetics of capsinoids in healthy male volunteers after a single oral ingestion of $\mathrm{CH}-19$ Sweet extract. Int J Toxicol. 2008;27 Suppl 3:137-47.

151. Kohlert C, Schindler G, Marz RW, Abel G, Brinkhaus B, Derendorf H, Grafe EU, Veit M. Systemic availability and pharmacokinetics of thymol in humans. J Clin Pharmacol. 2002;427:731-7.

152. Kanai M, Imaizumi A, Otsuka Y, Sasaki H, Hashiguchi M, Tsujiko K, Matsumoto S, Ishiguro H, Chiba T. Dose-escalation and pharmacokinetic study of nanoparticle curcumin, a potential anticancer agent with improved bioavailability, in healthy human volunteers. Cancer Chemother Pharmacol. 2012:691:65-70

153. Kanai M, Otsuka Y, Otsuka K, Sato M, Nishimura T, Mori Y, Kawaguchi M, Hatano E, Kodama Y, Matsumoto S, et al. A phase I study investigating the safety and pharmacokinetics of highly bioavailable curcumin (Theracurmin) in cancer patients. Cancer Chemother Pharmacol. 2013;716:1521-30.

154. Latimer B, Ekshyyan O, Nathan N, Moore-Medlin T, Rong X, Ma X, Khandelwal A, Christy HT, Abreo F, McClure G, et al. Enhanced systemic bioavailability of curcumin through transmucosal administration of a novel microgranular formulation. Anticancer Res. 2015;3512:6411-8.

Ready to submit your research? Choose BMC and benefit from:

- fast, convenient online submission

- thorough peer review by experienced researchers in your field

- rapid publication on acceptance

- support for research data, including large and complex data types

- gold Open Access which fosters wider collaboration and increased citations

- maximum visibility for your research: over $100 \mathrm{M}$ website views per year

At BMC, research is always in progress.

Learn more biomedcentral.com/submissions 